\title{
A chemical model for the atmosphere of hot Jupiters ${ }^{\star}$
}

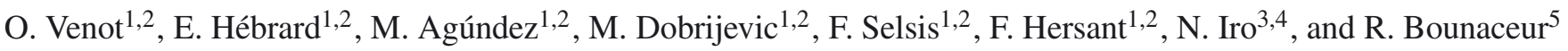 \\ 1 Univ. Bordeaux, LAB, UMR 5804, 33270 Floirac, France \\ e-mail: venot@obs.u-bordeaux 1 . fr \\ 2 CNRS, LAB, UMR 5804, 33270 Floirac, France \\ 3 Theoretical Meteorology group, Klimacampus, University of Hamburg, Grindelberg 5, 20144 Hamburg, Germany \\ ${ }^{4}$ LESIA, Observatoire de Paris, 5 Place Jules Janssen, 92195 Meudon, France \\ 5 Laboratoire Réactions et Génie des Procédés, LRGP UPR 3349 CNRS, Université de Lorraine, 1 rue Grandville, BP 20401, \\ 54001 Nancy, France
}

Received 29 March 2012 / Accepted 1 August 2012

\begin{abstract}
Context. The atmosphere of hot Jupiters can be probed by primary transit and secondary eclipse spectroscopy. Owing to the intense UV irradiation, mixing, and circulation, their chemical composition is maintained out of equilibrium and must be modeled with kinetic models.

Aims. Our purpose is to release a chemical network and the associated rate coefficients, developed for the temperature and pressure range relevant to hot Jupiters atmospheres. Using this network, we study the vertical atmospheric composition of the two hot Jupiters (HD 209458b and HD 189733b) with a model that includes photolyses and vertical mixing, and we produce synthetic spectra.

Methods. The chemical scheme has been derived from applied combustion models that were methodically validated over a range of temperatures and pressures typical of the atmospheric layers influencing the observations of hot Jupiters. We compared the predictions obtained from this scheme with equilibrium calculations, with different schemes available in the literature that contain N-bearing species, and with previously published photochemical models.

Results. Compared to other chemical schemes that were not subjected to the same systematic validation, we find significant differences whenever nonequilibrium processes take place (photodissociations or vertical mixing). The deviations from the equilibrium, hence the sensitivity to the network, are larger for HD 189733b, since we assume a cooler atmosphere than for HD 209458b. We found that the abundances of $\mathrm{NH}_{3}$ and $\mathrm{HCN}$ can vary by two orders of magnitude depending on the network, demonstrating the importance of comprehensive experimental validation. A spectral feature of $\mathrm{NH}_{3}$ at $10.5 \mu \mathrm{m}$ is sensitive to these abundance variations and thus to the chemical scheme.

Conclusions. Due to the influence of the kinetics, we recommend using a validated scheme to model the chemistry of exoplanet atmospheres. The network we release is robust for temperatures within 300-2500 K and pressures from 10 mbar up to a few hundred bars, for species made of $\mathrm{C}, \mathrm{H}, \mathrm{O}$, and $\mathrm{N}$. It is validated for species up to 2 carbon atoms and for the main nitrogen species $\left(\mathrm{NH}_{3}, \mathrm{HCN}\right.$, $\mathrm{N}_{2}, \mathrm{NO}_{x}$ ). Although the influence of the kinetic scheme on the hot Jupiters spectra remains within the current observational error bars (with the exception of $\mathrm{NH}_{3}$ ), it will become more important for atmospheres that are cooler or subjected to higher UV fluxes, because they depart more from equilibrium.
\end{abstract}

Key words. astrochemistry - planets and satellites: atmospheres - planets and satellites: individual: HD 209458b planets and satellites: individual: HD $189733 \mathrm{~b}$ - planetary systems

\section{Introduction}

So far, more than 700 exoplanets have been confirmed and thousands of transiting candidates identified by the space telescope Kepler (Batalha et al. 2012). Among them, hot Jupiters are a class of gas giants with orbital periods of a few days or less. They are found around $\sim 0.5 \%$ of KGF stars (Howard et al. 2010, 2012). About $10 \%$ of them transit their host star, and their atmospheric composition and physical structure can be studied by transit spectroscopy (e.g. Charbonneau et al. 2000, 2008; Richardson et al. 2007; Tinetti et al. 2007; Sing et al. 2008; Swain et al. 2008a,b, 2009a,b; Huitson et al. 2012).

Although current observations are still limited and subjected to divergent interpretations, future instruments such as E-ELT, JWST (Gardner et al. 2006), EChO (Tinetti et al. 2012), and

\footnotetext{
* Appendices are available in electronic form at http: //wWw . aanda.org
}

FINESSE (Swain 2010) should provide better constraints on both the chemical composition and the temperature profiles of the nearby hot Jupiters like HD 189733b and HD 209458b. They will also be able to study more distant targets and deliver statistically significant trends about the nature of their atmospheres. Chemical modeling will be an important component of these studies. It will point to key observations able to distinguish between various hypotheses and will be used to analyze the observations and to constrain, for instance, the atmospheric elemental abundances.

The first models of hot Jupiter atmospheres assumed chemical equilibrium (e.g. Burrows \& Sharp 1999; Seager \& Sasselov 2000; Sharp \& Burrows 2007; Barman 2007; Burrows et al. 2007, 2008; Fortney et al. 2008a). However, strongly irradiated atmospheres are unlikely to be at chemical equilibrium. Their intense UV irradiation (typically 10000 times the flux received on the top of the atmosphere of Jupiter) and strong dynamics result in photolyses and diffusion/advection timescales that 
are comparable to or shorter than the chemical ones. Deviations from the thermodynamic equilibrium have been discussed with timescale arguments (e.g. Lodders \& Fegley 2002; Fortney et al. 2006, 2008b; Visscher et al. 2006, 2010; Madhusudhan \& Seager 2010) or modeled with a few reactions describing the $\mathrm{CO}-\mathrm{CH}_{4}$ conversion coupled with the dynamics (Cooper \& Showman 2006). A more detailed modeling requires the use of a photochemical kinetic network. A kinetic network is, in practice, a list of reactions and associated rate coefficients able to quantitatively describe (within a certain accuracy) the kinetics of a pool of species, usually the most abundant ones. Constructing such a network of reactions implies answering two major questions. One has to do with the completeness of the network: what are the species and the reactions connecting them that must be included? The other issue is the availability of the kinetic data, because the literature and databases may not provide the rate coefficients for some of the needed reactions or may provide conflicting values with no recommendation. These two issues are tightly connected and both depend on the considered range of temperatures and pressures. Eventually, and regardless of the methodology adopted to select the reactions and their rates, it is the ability to predict experimentally-controled abundances that can validate the network or not.

To investigate the consequences of the strong UV incident flux on neutral species, photochemical models have been developed (Liang et al. 2003, 2004). Based on kinetics model dedicated to Jupiter's low-temperature atmosphere, these models however neglected endothermic reactions, which are in fact fairly efficient in such hot atmospheres. Line et al. (2010) have introduced some endothermic reactions to a similar Jovian photochemical scheme but most of the pre-existing reactions were not reversed. They were therefore not able to reproduce the thermodynamic equilibrium, which occurs in the deep atmospheres of hot Jupiters. Zahnle et al. (2009a,b) developed a photochemical model that considers the reversal of their whole set of twobody exothermic reactions. They selected their rate constants in the NIST database ${ }^{1}$ based on the following criteria: relevance of temperature conditions, date of review, date of the experiment, and date of the theoretical study (in order of preference). Moses et al. (2011) developed a model that considered the reversal of all the reactions, including three-body reactions, ensuring reproduction of the thermodynamical equilibrium from the top to the deepest layers of the atmosphere. Their chemical scheme is derived from the Jupiter and Saturn models (Gladstone et al. 1996; Moses 1996; Moses et al. 1995b,a, 2000a,b) with further updates on the basis of combustion-chemistry literature. In the same manner, Line et al. (2011) developed a fully reversible kinetic model, to study the hot Neptune GJ 436b. None of these works discuss the validation of the chemical scheme against experiments. In addition, that computed abundances evolve towards the composition predicted by equilibrium calculations (at given pressure $P$ and temperature $T$ with no external irradiation or mixing) is by no means a validation of the kinetic network. Indeed, any network containing at least as many independent, reversible reactions as modeled species, in which the rates for the backward processes are derived from equilibrium constants and forward rates, will evolve toward the equilibrium predicted with the same equilibrium constants, regardless of the quantitative values of the forward rates, as illustrated in Fig. 1. Fortunately, and thanks to the physical conditions and elemental composition of hot Jupiter (and hot Neptune) atmospheres, we benefit from decades of intensive work in the field of combustion,

\footnotetext{
${ }^{1}$ http://www.kinetics.nist.gov/kinetics
}

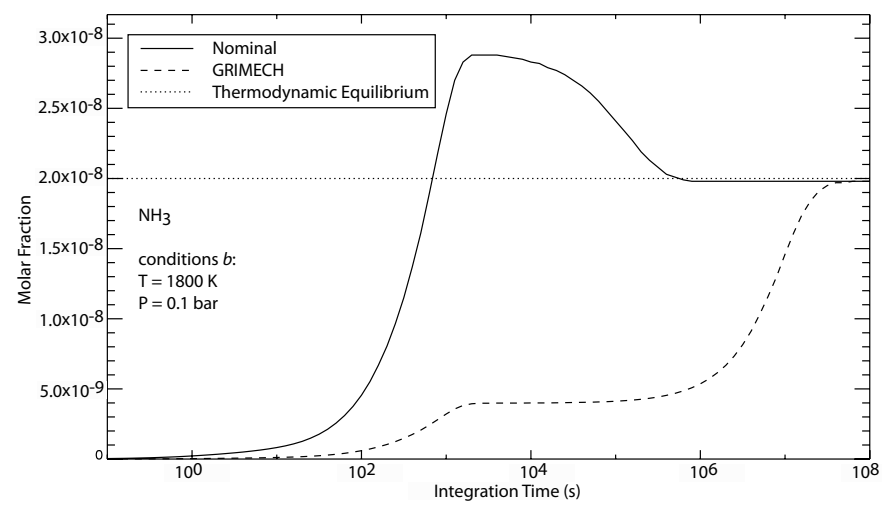

Fig. 1. Abundances of $\mathrm{NH}_{3}$ as a function of time computed with two kinetic schemes that are fully reversed according to equilibrium constants but that differs by their nitrogen chemistry (nominal and GRIMECH as defined in Sects. 2.1.2 and 3.3). While they both converge towards the equilibrium (dotted line), they exhibit very different evolution. Initial condition is a mixture of $\mathrm{H}_{2}, \mathrm{CH}_{4}, \mathrm{O}_{2}, \mathrm{~N}_{2}$, and $\mathrm{He}$ with solar elemental abundances.

which includes a vast amount of experiments, the development of comprehensive mechanisms ${ }^{2}$, and the systematic comparison between the two. Therefore, we propose in the present work a new mechanism dedicated to the chemical modeling of hot atmospheres that is not adapted from previous Solar System photochemical models but instead derives from industrial applications (mainly combustion in car engines). Details about this chemical network and its range of validity are presented in Sect. 2.1.

We use this chemical network in a 1D model that includes photolyses and vertical transport, which has been previously used to study the atmospheric photochemistry of various objects in the Solar System: Neptune (Dobrijevic et al. 2010a), Titan (Hébrard et al. 2006, 2007), Saturn (Dobrijevic et al. 2003; Cavalié et al. 2009), and Jupiter (Cavalié et al. 2008) as well as extrasolar terrestrial planets (Selsis et al. 2002). We model the photochemistry of two hot Jupiters: HD 209458b and HD 189733 b (Sect. 2.5). We study the departure from thermodynamic equilibrium and compare our results with those of Moses et al. (2011) (Sect. 3). We also investigate how including different reaction networks specific to nitrogen-bearing species influences the model results (Sect. 3.3) and the planetary synthetic spectra (Sect. 3.3.1).

\section{The model}

\subsection{Kinetic network: from car engine to hot Jupiters}

Significant progress has been made during the past decade in the development of validated combustion mechanisms. In the context of limiting the environmental impact of transportation, there is indeed a need for developing detailed chemical kinetic models that are more predictive and more accurate for the combustion of fuels. One part of the studies undertaken in the LRGP (Laboratoire Réactions et Génie des Procédés, Nancy, France) concerns engine-fuel adaptation to improve the efficiency of engines and to limit the emission of pollutants. Gasoline and diesel

\footnotetext{
2 In the field of combustion, a mechanism or reaction base is a network of reactions able to describe the kinetic evolution of a given pool of species. The mechanism includes the list of reactions and the associated rate coefficients, in a modified Arrhenius form, as well as the thermodynamic data for all the species involved in these reactions, which are required to calculate the equilibrium constants of the reactions and the rates of the reverse reactions.
} 
fuels contain many molecules belonging to several major hydrocarbon families. Biofuels contain also oxygenated species, such as alcohols and methyl esters. Oxidation and combustion of these complex blends occur by radical chain reactions involving hundreds of molecular and radical species and several thousand elementary reactions in the case of pure reference fuels, such as n-heptane, iso-octane, or cetane. The primary focus of the currently developed chemical models is to simulate the main combustion parameters (auto-ignition delay times, laminar flame speed, heat release), which are needed for the design of engines or burners, to estimate the fuel consumption, and to model the formation of some of the main regulated pollutants (carbon monoxide, nitrogen oxides, unburned hydrocarbons, and particulate matter). Most of these kinetic models were developed for industrial applications and have been validated in a range of temperatures, from 300 to approximately $2500 \mathrm{~K}$, and for pressures from 0.01 bar to some hundred bar. What is worth noticing is the similarity of these temperature and pressure ranges with the conditions prevailing in hot Jupiters atmospheres, in the very layers where they influence the observed molecular features. In addition, combustion mechanisms mainly deal with molecules made of $\mathrm{C}, \mathrm{H}, \mathrm{O}$, and $\mathrm{N}$, which are also the main constituents of the molecules and radicals in these atmospheres. For this reason, we have decided to implement such a mechanism, which has already been applied successfully to many cases and systematically validated (Bounaceur et al. 2007), to study the atmosphere of hot Jupiters.

In this study we have used a $\mathrm{C} / \mathrm{H} / \mathrm{O} / \mathrm{N}$ mechanism, whose core is a $\mathrm{C}_{0}-\mathrm{C}_{2}$ mechanism that includes all the reactions required to model the kinetic evolution of radicals and molecules containing fewer than three carbon atoms. This mechanism also contains some species with more than two carbon atoms, which are necessary to model the abundance of $\mathrm{C}_{0}-\mathrm{C}_{2}$ species. This mechanism does not include nitrogen species, except $\mathrm{N}_{2}$ as a third body. Because nitrogen species, such as $\mathrm{N}_{2}, \mathrm{NH}_{3}, \mathrm{HCN}$, and $\mathrm{CN}$, are expected to be important constituents of hot Jupiter atmospheres, we completed this $\mathrm{C}_{0}-\mathrm{C}_{2}$ base with a validated submechanism specifically constructed to model nitrogen species and all the cross-term reactions involved (for instance, reactions between alkanes and $\mathrm{NO}_{x}$ ). These mechanisms do not use rate coefficients that have been adjusted by optimization procedures in order to fit experiments. Their values are those recommended for the individual processes by the main kinetics databases for combustion (Tsang \& Hampson 1986; Manion et al. 2008; Smith et al. 1999; Baulch et al. 2005). The list of the reactions and their rate coefficients are available in the online database KIDA: KInetic Database for Astrochemistry ${ }^{3}$ (Wakelam et al. 2012). The final mechanism includes 957 reversible and 6 irreversible reactions (see Sect. 2.1.3), involving 105 neutral species (molecule or radical). Helium is also included in this mechanism and plays the role of third body in some reactions.

\subsection{1. $\mathrm{C}_{0}-\mathrm{C}_{2}$ reaction base}

The $\mathrm{C}_{0}-\mathrm{C}_{2}$ reaction base we use was developed for industrial applications, was first presented by Barbé et al. (1995), and has been continuously updated (Fournet et al. 1999; Bounaceur et al. 2010). This mechanism is designed to reproduce the kinetics of species with fewer than three carbons. It includes all the unimolecular or bimolecular reactions involving radicals or molecules containing no more than two carbon atoms.

\footnotetext{
${ }^{3}$ http://kida.obs.u-bordeaux1.fr
}

This mechanism was built by using a reaction grid, as proposed by Tsang \& Hampson (1986). All unimolecular and bimolecular elementary forward reactions involving the considered reacting species were systematically written. Reacting species include 46 compounds (19 molecules and 27 radicals), which were ranked according to the molecular formula $\mathrm{O}_{x} \mathrm{C}_{y} \mathrm{H}_{z}$ (with $x$ varying from 0 to $3, y$ from 0 to 2 , and $z$ from 0 to 6): $\mathrm{CO}, \mathrm{H}_{2}, \mathrm{H}_{2} \mathrm{O}, \mathrm{O}_{2}, \mathrm{H}_{2} \mathrm{O}_{2}, \mathrm{CH}_{4}, \mathrm{H}_{2} \mathrm{CO}, \mathrm{CH}_{3} \mathrm{OH}, \mathrm{CO}_{2}$, $\mathrm{CH}_{3} \mathrm{OOH}, \mathrm{C}_{2} \mathrm{H}_{2}, \mathrm{C}_{2} \mathrm{H}_{4}, \mathrm{C}_{2} \mathrm{H}_{6}, \mathrm{CH}_{2} \mathrm{CO}, \mathrm{CH}_{3} \mathrm{CHO}, \mathrm{C}_{2} \mathrm{H}_{5} \mathrm{OH}$, $\mathrm{C}_{2} \mathrm{H}_{5} \mathrm{OOH}, \mathrm{CH}_{3} \mathrm{COOOH}, \mathrm{cC}_{2} \mathrm{H}_{4} \mathrm{O}$ (Ethylene Oxide), $\mathrm{C}, \mathrm{CH}$, ${ }^{1} \mathrm{CH}_{2}$ (singlet), ${ }^{3} \mathrm{CH}_{2}$ (triplet), $\mathrm{O}\left({ }^{3} \mathrm{P}\right), \mathrm{H}, \mathrm{OH}, \mathrm{OOH}, \mathrm{CH}_{3}$, $\mathrm{HCO}, \mathrm{CH}_{2} \mathrm{OH}, \mathrm{CH}_{3} \mathrm{O}, \mathrm{CH}_{3} \mathrm{OO}, \mathrm{C}_{2} \mathrm{H}, \mathrm{C}_{2} \mathrm{H}_{3}, \mathrm{C}_{2} \mathrm{H}_{5}, \mathrm{CHCO}$, $\mathrm{CH}_{2} \mathrm{CHO}, \mathrm{CH}_{3} \mathrm{CO}, \mathrm{C}_{2} \mathrm{H}_{5} \mathrm{O}, \mathrm{C}_{2} \mathrm{H}_{4} \mathrm{OOH}, \mathrm{C}_{2} \mathrm{H}_{5} \mathrm{OO}, \mathrm{CH}_{3} \mathrm{COOO}$, $\mathrm{CH}_{3} \mathrm{OCO}, \mathrm{CO}_{2} \mathrm{H}, 1-\mathrm{C}_{2} \mathrm{H}_{4} \mathrm{OH}$ and $2-\mathrm{C}_{2} \mathrm{H}_{4} \mathrm{OH}$ (ethyl radical isomers, 1-hydroxy, and 2-hydroxy). This ranking permits the part of the mechanism related to pyrolysis reactions to be separated easily from the one related to oxidation or combustion. The mechanism also includes 14 species containing three or four carbon atoms: $\mathrm{C}_{3} \mathrm{H}_{8}, \mathrm{C}_{4} \mathrm{H}_{8}, \mathrm{C}_{4} \mathrm{H}_{10}, \mathrm{C}_{2} \mathrm{H}_{5} \mathrm{CHO}, \mathrm{C}_{3} \mathrm{H}_{7} \mathrm{OH}, \mathrm{C}_{3} \mathrm{H}_{7} \mathrm{O}$, $\mathrm{C}_{4} \mathrm{H}_{9} \mathrm{O}, \quad \mathrm{C}_{2} \mathrm{H}_{6} \mathrm{CO}, \mathrm{C}_{3} \mathrm{H}_{8} \mathrm{CO}, \mathrm{C}_{2} \mathrm{H}_{3} \mathrm{CHO}, \quad \mathrm{n}-\mathrm{C}_{3} \mathrm{H}_{7}, \quad \mathrm{i}-\mathrm{C}_{3} \mathrm{H}_{7}$ (isopropyl and n-propyl radical isomers), $1-\mathrm{C}_{4} \mathrm{H}_{9}$ and $2-\mathrm{C}_{4} \mathrm{H}_{9}$ (1-butyl and 2-butyl radical isomers).

This $\mathrm{C}_{0}-\mathrm{C}_{2}$ mechanism has been widely validated in the 300-2500 K, 0.01-100 bar range for several types of reactors, such as shock-tubes, perfectly stirred reactors, plug-flow reactors, rapid compression machines, and laminar flames (e.g. Battin-Leclerc et al. 2006; Bounaceur et al. 2007; Anderlohr et al. 2010; Bounaceur et al. 2010; Wang et al. 2010). Obviously, it is not possible to describe all these validations in detail, but we can mention, for instance, the very recent work of Dirrenberger et al. (2011) who has studied the laminar burning velocity of several mixtures including air, hydrogen, and components of natural gas experimentally and modeled it with success. Laminar burning velocities are important parameters in many areas of combustion science, such as the design of burners and the prediction of explosions. They also play an essential role in determining several important aspects of the combustion process in spark ignition engines. These experiments were done in specific mixtures, containing nitrogen in the sole form of $\mathrm{N}_{2}$ and in which nitrogen species produced from $\mathrm{N}_{2}\left(\mathrm{NO}_{x}\right.$ in the typical mixtures used in combustion) do not significantly affect the outcome, in order to validate the $\mathrm{C}_{0}-\mathrm{C}_{2}$ mechanism itself. Therefore, a model including only the $\mathrm{C}_{0}-\mathrm{C}_{2}$ base would not be accurate to predict the abundance of $\mathrm{C}_{0}-\mathrm{C}_{2}$ species in this range of $P$ and $T$ when applied to mixtures containing or producing (by reaction with $\mathrm{N}_{2}$ ) significant levels of nitrogen species other than $\mathrm{N}_{2}$.

\subsubsection{Nitrogen reaction base}

In our nominal model, the subnetwork for the nitrogen bearing species was derived from Konnov (2000, 2009) and Coppens et al. (2007). It is based on a comprehensive analysis of the combustion chemistry of nitrogen oxides (Konnov \& De Ruyck 1999a), ammonia (Konnov \& De Ruyck 2000b), hydrazine (Konnov \& De Ruyck 2001b), and modeling of nitrogen oxides formation in different combustion systems (Konnov \& De Ruyck 1999b, 2000a, 2001a). The mechanism was tested at the California Institute of Technology, USA, and found to be suitable for steady one-dimensional detonation and constant volume explosion simulations (Schultz \& Shepherd 1999). It was also preferred by the researchers at the University of Bielefeld, Germany, to analyze flame structure and NO reburning in $\mathrm{C}_{3}$ flames (Atakan \& Hartlieb 2000). In addition, we 
consider a few additional pathways for $\mathrm{HCN}$ oxidation from Dagaut et al. (2008).

Validations of our nominal subnetwork for nitrogen-bearing species were made on the basis of experimental data obtained, for instance, by oxidation of $\mathrm{HCN}$ in a silica jet-stirred reactor (JSR) at atmospheric pressure and from 1000 to $1400 \mathrm{~K}$ (Dagaut et al. 2008), or by studying laminar flame speeds in $\mathrm{NH}_{3}-\mathrm{N}_{2} \mathrm{O}$ mixtures (Brown \& Smith 1994). The nitrogen mechanism includes 42 species (molecule or radical): $\mathrm{NO}_{3}, \mathrm{HONO}_{2}$, $\mathrm{CH}_{3} \mathrm{ONO}, \mathrm{CH}_{3} \mathrm{NO}_{2}, \mathrm{HNO}_{2}, \mathrm{CH}_{3} \mathrm{NO}, \mathrm{NO}_{2}, \mathrm{HONO}, \mathrm{HCNN}$, $\mathrm{HCNO}, \mathrm{N}_{2} \mathrm{O}, \mathrm{NCO}, \mathrm{HNO}, \mathrm{HOCN}, \mathrm{NNH}, \mathrm{H}_{2} \mathrm{CN}, \mathrm{N}\left({ }^{4} \mathrm{~S}\right), \mathrm{CN}$, HNCO, NO, NH, $\mathrm{NH}_{2}, \mathrm{HCN}, \mathrm{NH}_{3}, \mathrm{~N}_{2}, \mathrm{~N}_{2} \mathrm{O}_{4}, \mathrm{~N}_{2} \mathrm{O}_{3}, \mathrm{~N}_{2} \mathrm{H}_{2}$, $\mathrm{N}_{2} \mathrm{H}_{3}, \mathrm{~N}_{2} \mathrm{H}_{4}, \mathrm{HNNO}, \mathrm{HNOH}, \mathrm{HNO}_{3}, \mathrm{NH}_{2} \mathrm{OH}, \mathrm{H}_{2} \mathrm{NO}, \mathrm{CNN}$, $\mathrm{H}_{2} \mathrm{CNO}, \mathrm{C}_{2} \mathrm{~N}_{2}, \mathrm{HCNH}, \mathrm{HNC}, \mathrm{HON}$ and NCN.

For comparison, we also used other nitrogen submechanisms, which are presented in Sect. 3.3 with the corresponding results.

Because the mechanism we use was created from individual processes and validated without any optimization of their reaction coefficients, its application outside the condition range of validation is not problematic. This is a problem, for instance, with the well-known combustion mechanism GRI-Mech 3.04 (Smith et al. 1999), proposed by Gas Research Institute, which is an optimized mechanism designed to model natural gas combustion. Optimization makes the model extremely accurate within the optimization domain, but its application beyond is risky (Battin-Leclerc et al. 2011).

\subsubsection{Reversible reactions: kinetics vs. thermodynamics}

For most reversible reactions, rate coefficients are only available for the exothermic (forward) direction. The rate constant for the endothermic (reverse) direction, $k_{\mathrm{r}}(T)$, is then calculated as the ratio between the forward rate constant $k_{\mathrm{f}}(T)$ and the equilibrium constant $K_{\mathrm{eq}}(T)$, calculated with thermodynamical data, as explained in Appendix A. However, rate coefficients have sometimes been measured for both directions. In such cases, the ratio $k_{\mathrm{f}}(T) / k_{\mathrm{r}}(T)$ departs from $K_{\text {eq }}(T)$ because different uncertainties affect the rate coefficients and the thermodynamic data. The computation of $k_{\mathrm{r}}(T)$ using $K_{\text {eq }}(T)$ ensures the consistency between kinetics and thermodynamics by making the kinetic model evolve strictly toward the thermodynamic equilibrium that we calculate. Nevertheless, this choice may not always be the best. It results in the propagation into $k_{\mathrm{r}}(T)$ of both the errors affecting $k_{\mathrm{f}}(T)$ and $K_{\text {eq }}(T)$. Indeed, thermodynamic parameters used to calculate $K_{\mathrm{eq}}(T)$ are not free of error, and are regularly updated just as kinetic data. In the field of combustion, for small species, such as $\mathrm{CH}_{4}, \mathrm{CH}_{3}$, and $\mathrm{OH}$, it is common to use experimentally measured kinetic rates, rather than thermodynamical reversal, when they are available in the relevant temperature range. There is no obvious rule in this matter, but validation of the mechanism with out-of-equilibrium experiments seems the only practical way to chose between different rates. This is the criterion that we use, and our nominal network uses thermodynamical reversal for most of the reactions but not for three important ones. These reactions affect the unimolecular initiations (or thermal dissociation reactions) of methane into methyl and hydrogen radicals,

$$
\mathrm{CH}_{4} \stackrel{M}{\rightarrow} \mathrm{CH}_{3}+\mathrm{H}
$$

\footnotetext{
${ }^{4}$ http://www.me.berkeley.edu/gri_mech/
}

of ethane into two methyl radicals,

$\mathrm{C}_{2} \mathrm{H}_{6} \stackrel{M}{\longrightarrow} \mathrm{CH}_{3}+\mathrm{CH}_{3}$,

and of hydrogen peroxide into two hydroxyl radicals,

$\mathrm{H}_{2} \mathrm{O}_{2} \stackrel{M}{\rightarrow} \mathrm{OH}+\mathrm{OH}$.

At high temperature, chemical kinetics is very sensitive to these three reactions, which have been widely studied experimentally (Baulch et al. 1994; Golden 2008; Troe 2011). Therefore, we use the kinetic data measured experimentally for the forward and the reverse directions instead of calculating the reverse rate constants using thermodynamic parameters.

\subsubsection{Excitation of oxygen and nitrogen atoms}

Photodissociations produce excited states of oxygen $\left(\mathrm{O}\left({ }^{1} \mathrm{D}\right)\right)$ and nitrogen $\left(\mathrm{N}\left({ }^{2} \mathrm{D}\right)\right)$ that are not treated in the original combustion mechanisms. Therefore, we added 19 reversible reactions to the $\mathrm{C} / \mathrm{H} / \mathrm{O} / \mathrm{N}$ mechanism, which describe the kinetics of $\mathrm{O}\left({ }^{1} \mathrm{D}\right)$ and $\mathrm{N}\left({ }^{2} \mathrm{D}\right)$, including radiative and collisional desexcitation. These reactions rates are taken (or have been estimated) from Okabe (1978), Herron (1999), Umemoto et al. (1998), Balucani et al. (2000a), Sato et al. (1999), Balucani et al. (2000b), and Sander et al. (2011).

\subsection{Test of the chemical scheme with a OD model}

In addition to our 1D model, we have also developed a simple OD model that computes the chemical evolution of a mixture at constant temperature and pressure. It includes neither mixing with another mixture nor photolyses. We used this OD model to compare the composition found at steady state with the abundances at thermodynamic equilibrium (calculated with the code TECA, described in Appendix B) for several couples of pressure-temperature. Figure 2 illustrates this comparison with four species. First, we used a version of our nominal scheme in which all the reactions are reversed, in agreement with their equilibrium constant. The computed abundances converge exactly towards the equilibrium values with negligible numerical differences. Then, we used our nominal model in which some reactions are not reversed according to their equilibrium constant but using rate coefficients measured experimentally. In this case, the abundances reached at steady state departs from the predicted equilibrium. This departure remains very small: below $1 \%$ for most species and always below $5 \%$. However, one can see that the kinetic evolution can be significantly different, both in terms of abundances and timescales.

\subsection{The $1 D$ model}

To model the chemical composition of the atmosphere of the hot Jupiters HD 209458b and HD 189733b, we use our 1D time-dependent model, described in Dobrijevic et al. (2010a). As an input of the model, we give a pressure-temperature profile for the atmosphere of the planet being studied. This profile is then divided into discrete uniform layers with a thickness $\Delta z=\frac{H(z)}{8}$, where $H(z)$ is the pressure scale height. The grid contains $\sim 300$ layers. Then the 1D kinetic model resolves the continuity equation (Eq. (4)) as a function of time, for each species and atmospheric layer, until a steady state is reached.

$\frac{\partial n_{i}}{\partial t}=P_{i}-n_{i} L_{i}-\operatorname{div}\left(\Phi_{i} \overrightarrow{e_{z}}\right)$ 
O. Venot et al.: A chemical model for the atmosphere of hot Jupiters
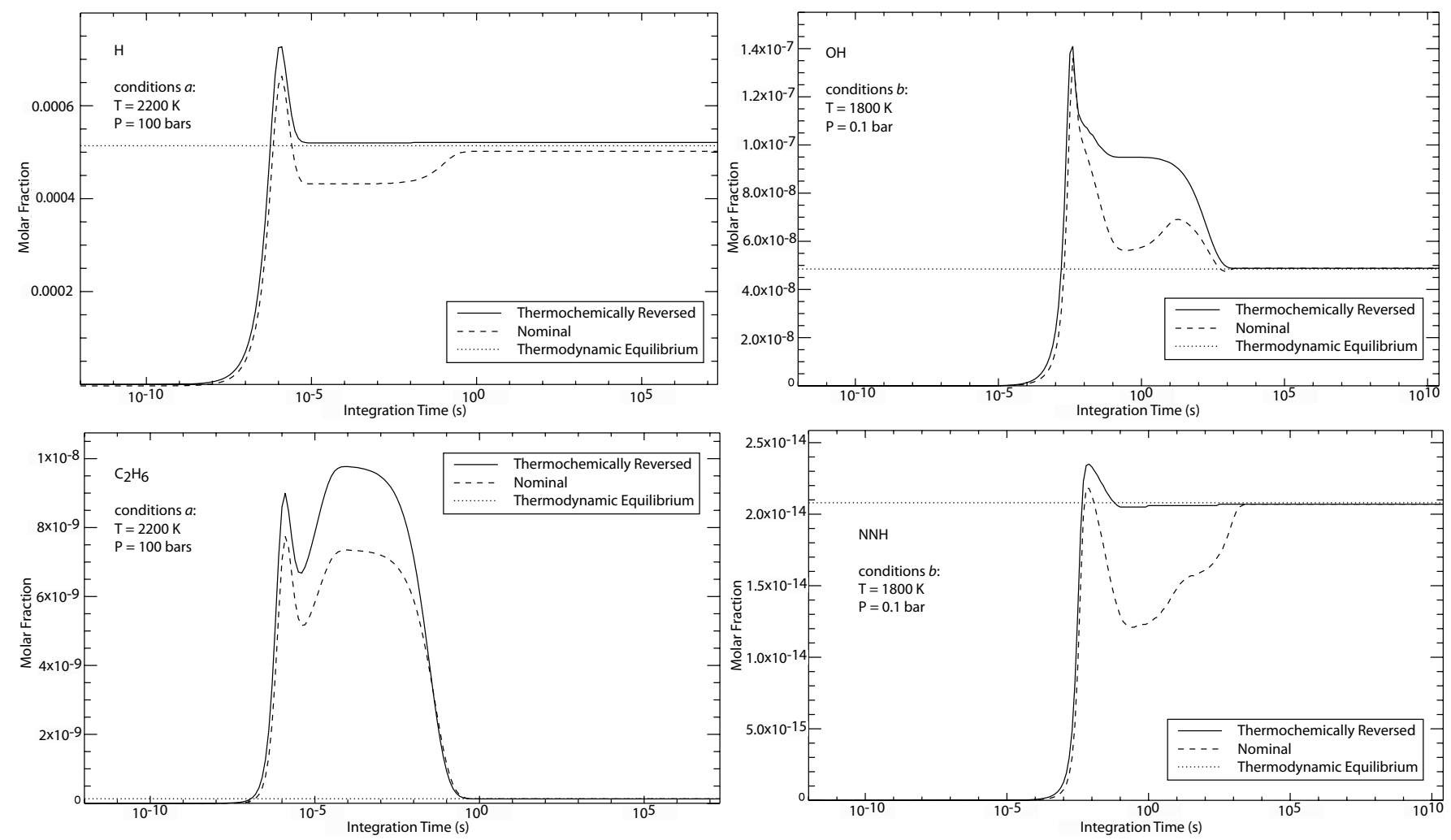

Fig. 2. Comparison between the thermodynamic equilibrium (dotted line) and the evolution of some molecular abundances in the 0D model, with two different schemes: the thermochemically reversed model (solid line) and the nominal model (dashed line) as a function of integration time at different temperature-pressure points. Initial condition is a mixture of $\mathrm{H}_{2}, \mathrm{CH}_{4}, \mathrm{O}_{2}, \mathrm{~N}_{2}$, and $\mathrm{He}$ with solar elemental abundances.

where $n_{i}$ the number density of the species $i\left(\mathrm{~cm}^{-3}\right), P_{i}$ its production rate $\left(\mathrm{cm}^{-3} \mathrm{~s}^{-1}\right), L_{i}$ its loss rate $\left(\mathrm{s}^{-1}\right)$, and $\Phi_{i}$ its vertical flux $\left(\mathrm{cm}^{-2} \mathrm{~s}^{-1}\right)$ that follows the diffusion equation,

$\Phi_{i}=-n_{i} D_{i}\left[\frac{1}{n_{i}} \frac{\partial n_{i}}{\partial z}+\frac{1}{H_{i}}+\frac{1}{T} \frac{\mathrm{d} T}{\mathrm{~d} z}\right]-n_{i} K\left[\frac{1}{y_{i}} \frac{\partial y_{i}}{\partial z}\right]$

where $K$ is the eddy diffusion coefficient $\left(\mathrm{cm}^{2} \mathrm{~s}^{-1}\right), D_{i}$ is the molecular diffusion coefficient $\left(\mathrm{cm}^{2} \mathrm{~s}^{-1}\right)$, and $H_{i}$ the scale height of the species $i$.

At both upper and lower boundaries, we impose a zero flux for each species.

\subsection{Photochemistry}

We add a set of 34 photodissociations to the thermochemical scheme, which are presented in Appendix D. As we can see in Fig. 3, for HD 209458b and HD 189733b, UV flux penetrates down to a pressure of about 1 bar, where the temperature is higher than $1500 \mathrm{~K}$. At these temperature and pressure, endothermic reactions do matter, which implies that photochemistry and thermochemistry are coupled in such highly irradiated atmospheres. We used absorption cross sections at the highest available temperature (i.e. $370 \mathrm{~K}$ at maximum, which is low compared to the temperatures in the atmosphere of hot Jupiters (see Fig. 4)).

To calculate the photodissociation rates in all the layers of the atmosphere, we computed the stellar UV flux as a function of pressure and wavelength, taking molecular absorption by 22 species (Appendix D) and Rayleigh scattering into account. Actinic fluxes are calculated with a resolution of $1 \mathrm{~nm}$ (which is also the resolution we adopted for the absorption cross-sections), assuming a plane parallel geometry and

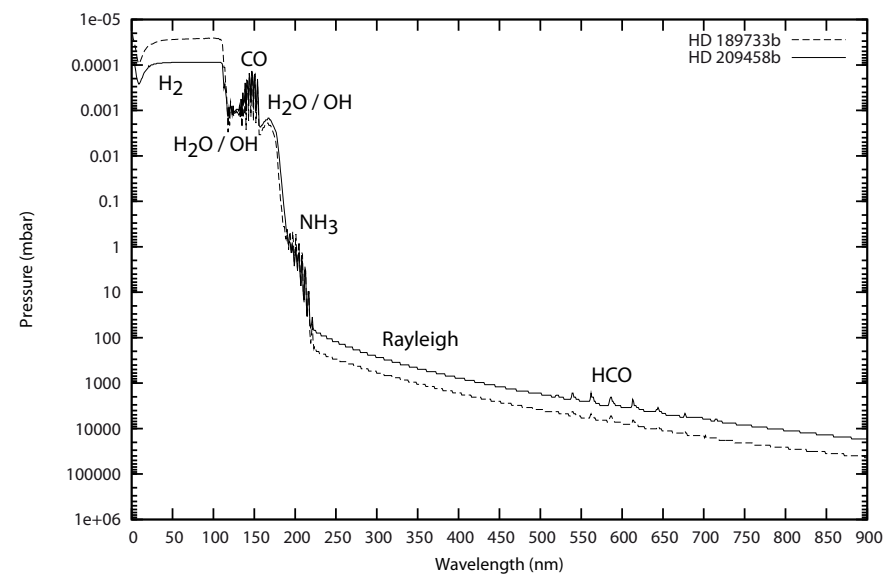

Fig. 3. Penetration of UV flux in the atmosphere of HD 209458b and HD 189733 b at the steady state in function of wavelength. Plots represent the level where the optical depth $\tau=1$. The name of the compounds responsible for the main absorption at different wavelengths is indicated.

an incidence angle $\theta$ of $48^{\circ}$ (as done in Moses et al. 2011, because $\langle\cos \theta\rangle=2 / 3\left(\theta \simeq 48^{\circ}\right)$ is the projected-area weighted average of the cosine of the stellar zenith angle over the planetary disk in secondary-eclipse conditions). Multiple Rayleigh scattering is coupled with absorption through a simple two-stream iterative algorithm (Isaksen et al. 1977).

\subsection{Application to hot Jupiters: HD 209458b and HD 189733b}

HD 209458b and HD 189733b are transiting planets around nearby bright stars. Their atmospheres have been studied by 

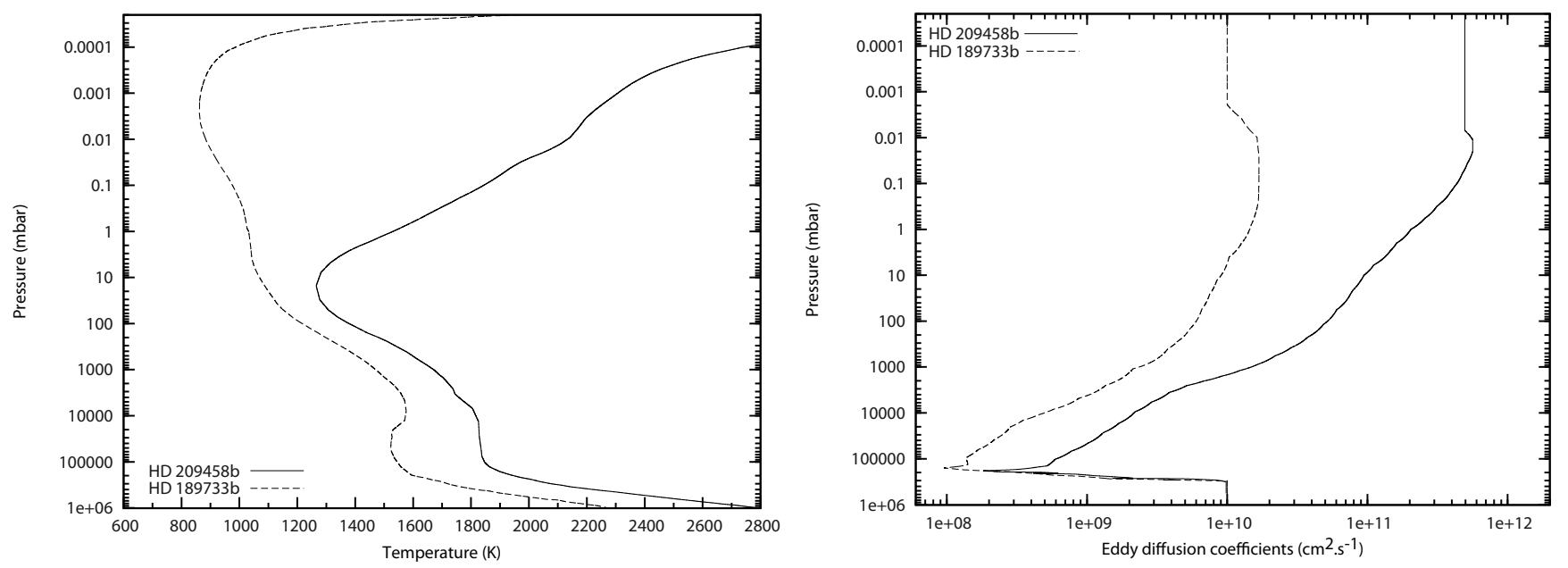

Fig. 4. Pressure-temperature profiles (left) and eddy diffusion profiles (right) of HD 189733b and HD 209458b (from Moses et al. 2011).

their transmission spectrum obtained during the primary transit and their day-side emission spectrum measured at the secondary eclipse. These observations can be used to constrain the thermal profile (Swain et al. 2009a; Madhusudhan \& Seager 2009) and to detect the spectral signature of atmospheric compounds (Charbonneau et al. 2002; Tinetti et al. 2007; Swain et al. 2008b; Grillmair et al. 2008; Langland-Shula et al. 2009; Swain et al. 2009a,b; Beaulieu et al. 2010).

In this preliminary study, we do not compare the results of our model with these observations for two reasons. First, there is no consensus yet on the actual constraints that can be drawn from these measurements. Secondly, such a comparison would imply addressing the effects of circulation on the composition and exploring all the range of possible elemental abundances for these objects. Several recent works claim that observations of some hot Jupiters imply enhanced elemental $\mathrm{C} / \mathrm{O}$ ratios (Madhusudhan et al. 2011a,b). With C/O ratios close or above unity, species with more than two carbon atoms will be important, and our $\mathrm{C}_{0}-\mathrm{C}_{2}$ network does not allow us to study them. For these reasons, we are implementing a $\mathrm{C}_{0}-\mathrm{C}_{6}$ mechanism and a coupling with atmospheric circulation, which will be described in further studies. At this stage, our main goal is to compare the results of our model with already published works, in particular Moses et al. (2011, hereafter M11). We could also have compared our results with those of Zahnle et al. (2009a), who explored a broader range of conditions, included sulfurbearing species and various elemental compositions. We decided to restrict our comparison with M11 because their model, like ours, only includes species made of $\mathrm{C}, \mathrm{H}, \mathrm{O}$, and $\mathrm{N}$ (and $\mathrm{He}$ ), and also because M11 have already made a comparison between their results and those of Zahnle et al. (2009a) showing only little discrepancies when the same conditions are considered. We used the same conditions ( $P-T$ profiles, eddy diffusion, elemental abundances) as in M11, so that differences should only come from kinetics (and photochemistry in the upper atmosphere), which represents the novelty of our approach.

\subsubsection{Physical properties and composition}

The physical properties of HD 209458b have been refined by Rowe et al. (2008) and are presented in Table 1, with some properties of the host star. Properties of HD 189733b and HD 189733 come from Southworth $(2008,2010)$.

To compare the outcomes of the two models (Sect. 3.2), we used the temperature and eddy diffusion profiles published
Table 1. Properties of the systems HD 209458 and HD 189733.

\begin{tabular}{lcc}
\hline \hline & HD 209458 & HD 189733 \\
\hline Distance Sun-Star $(\mathrm{pc})$ & 47 & 19.3 \\
Distance Planet-Star (AU) & 0.047 & 0.03142 \\
$M_{\mathrm{p}}\left(M_{\text {Jup }}\right)$ & $0.69 \pm 0.01$ & $1.150 \pm 0.028$ \\
$R_{\mathrm{p}}\left(R_{\text {Jup }}\right)$ & $1.339 \pm 0.002$ & $1.151 \pm 0.036$ \\
$\rho_{\mathrm{p}}\left(\rho_{\text {Jupiter }}\right)$ & $0.26 \pm 0.04$ & $0.755 \pm 0.066$ \\
$g_{0}\left(\mathrm{~m} \mathrm{~s}^{-2}\right)$ & $9.54 \pm 0.69$ & $21.5 \pm 1.2$ \\
$P_{\text {orbit }}($ days $)$ & $3.5247489(2)$ & $2.21857578(80)$ \\
$M_{\star}\left(M_{\odot}\right)$ & $1.083 \pm 0.005$ & $0.840 \pm 0.030$ \\
$R_{\star}\left(R_{\odot}\right)$ & $1.118 \pm 0.002$ & $0.752 \pm 0.023$ \\
Spectral type & G0 V & K1 V-K2 V \\
\hline
\end{tabular}

Notes. The $1 \sigma$ uncertainty in $P_{\text {orbit }}$ is given in parentheses in units of the last digits.

in M11 (Fig. 4). Also following M11, we assumed protosolar elemental abundances (Lodders \& Palme 2009) for both planets, with $20 \%$ of depletion for oxygen (sequestered along with silicates and metals). We started our time-dependent modeling with the thermodynamic equilibrium abundances calculated with TECA (an equilibrium model described in Appendix B) at each level of the atmosphere.

\subsubsection{UV spectral irradiance}

As HD 209458 is a G0 star (Table 1), we use the UV spectral irradiance of the Sun for this star. For the star HD 189733, a $\mathrm{K} 1-\mathrm{K} 2$ star (Table 1), the UV spectrum has been provided to us by Ignasi Ribas (priv. comm.). It is based on FUSE and HST observations of the star $\epsilon$ Eridani, a proxy of HD 189733 (similar type, age, and metallicity), in the $90-330 \mathrm{~nm}$ range. Between 0.5 and $90 \mathrm{~nm}$, it is based on data from the X-exoplanets Archive at the CAB (Sanz-Forcada et al. 2011). Above $330 \mathrm{~nm}$, we use a synthetic spectrum calculated with the stellar atmosphere code Phoenix (Hauschildt et al. 1999). This model for the UV spectrum of HD 189733 differs slightly from the one chosen in M11. We also tested our model with the spectrum used by M11 and found negligible differences at the pressure levels we model.

\section{Results}

\subsection{Nominal model}

First of all, we checked that our kinetic model reproduces the thermodynamic equilibrium, in the absence of vertical mixing 

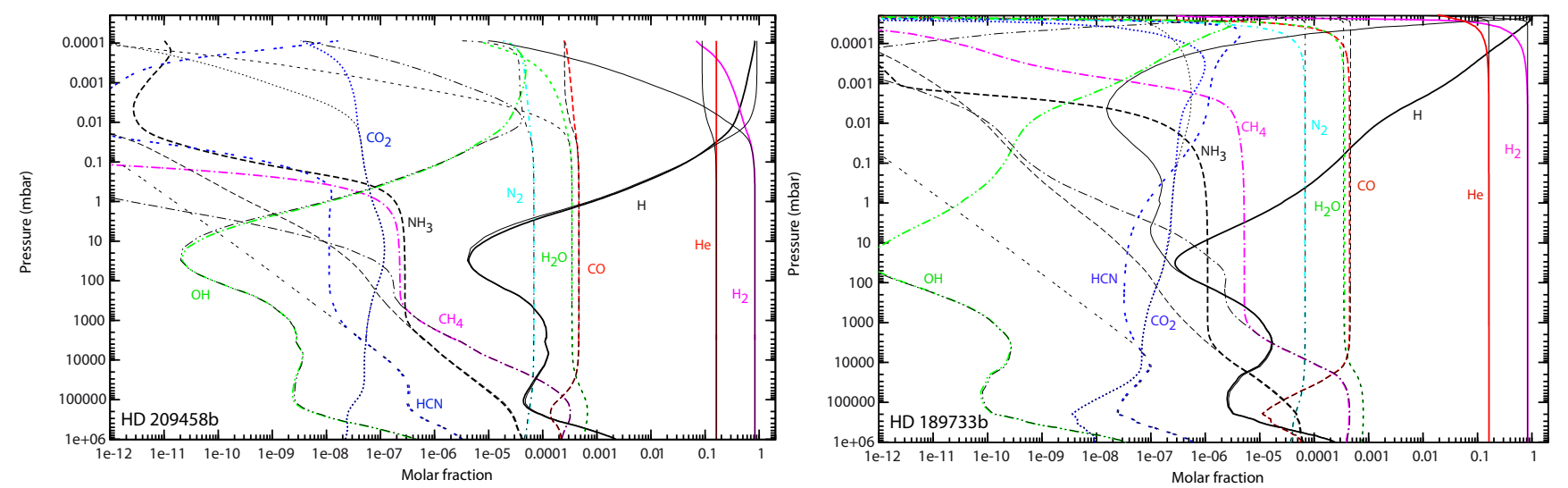

Fig. 5. Steady-state composition of HD 209458b (left) and HD 189733b (right) calculated with our nominal model (color lines), compared to the thermodynamic equilibrium (thin black lines).
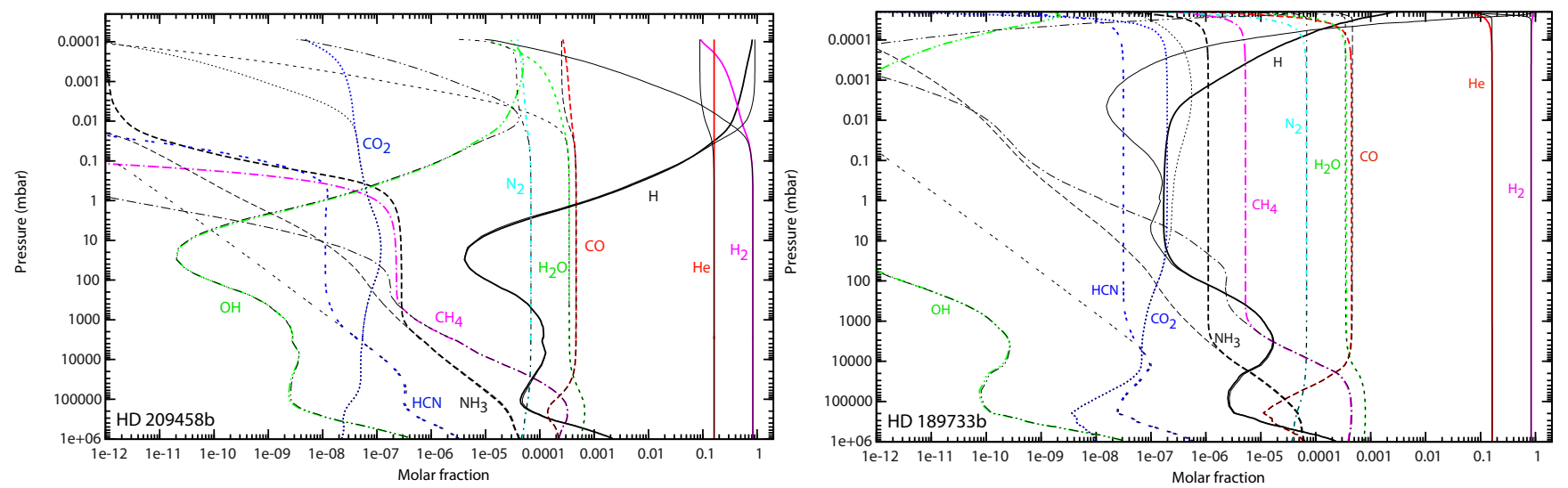

Fig. 6. Steady-state composition of HD 209458b (left) and HD 189733b (right) calculated with our nominal model without photodissociation (color lines), compared to the thermodynamic equilibrium (thin black lines).

and photodissociation. We obtained differences lower than a few percent, as found with the 0D model (see Sect. 2.2). For both planets, the homopause is always found above the $1 \times 10^{-5} \mathrm{mbar}$ level, which is beyond the range of pressure that we model. As a consequence, and although it is included, molecular diffusion does not affect our results.

Figure 5 shows the steady-state composition of the atmosphere of HD 209458b and HD 189733b, with vertical transport and photodissociations, while in Fig. 6, photodissociations have been removed. Comparing Figs. 5 and 6 shows us the influence of photolyses. Although HD 209458b receives a higher UV flux than HD 189733b, we can see that UV photons have little effect on the composition of HD 209458b, while they have significant influence on the chemistry of HD $189733 \mathrm{~b}$. This is because the temperature is higher in HD 209458b so that the chemical timescales are significantly shorter than the lifetime of species against photolyses, so in HD 209458b, kinetics dominate over photodissociations, even at high altitude. In HD 189733b, however, photodissociations affect the composition down to about the 10 mbar level. This is particularly noticeable for $\mathrm{H}$ and $\mathrm{OH}$ abundances. The production of $\mathrm{H}$ is dominated by the photolysis of $\mathrm{H}_{2}$ for pressures lower than $1 \mu \mathrm{bar}$. Below this level, and for pressures higher than 0.1 mbar, $\mathrm{H}$ is produced by the photodissociation of $\mathrm{H}_{2} \mathrm{O}$, with a minor contribution of the photodissociations of $\mathrm{NH}_{3}$ and $\mathrm{HCN}$. The abundance of $\mathrm{OH}$ follows the profile of $\mathrm{H}$, and increases for pressures lower than 10 mbar. There is a photochemical enhancement of HCN above the 10 mbar pressure level, as discussed in $\mathrm{M} 11 . \mathrm{CH}_{4}$ is destroyed by photolyses for pressures lower than 0.01 mbar.
$\mathrm{NH}_{3}$ is photodissociated down to levels as deep as 1 bar, but vertical transport compensates for this destruction for pressures higher than 0.1 mbar. Above that level, the amount of $\mathrm{NH}_{3}$ decreases with altitude due to photolyses. Photochemistry has a negligible effect on $\mathrm{CO}_{2}$, as noted by Zahnle et al. (2009a).

For HD 209458b, we can see in Fig. 6 that mixing quenches $\mathrm{NH}_{3}$ and $\mathrm{HCN}$ at 1 bar and $\mathrm{CH}_{4}$ at 400 mbar. These species are transported up to the $\sim 1$ mbar pressure level, but because the temperature increases with altitude at this level, they tend to come back to their thermochemical equilibrium values, so their abundances decrease again. For the other molecules, such as $\mathrm{H}_{2}, \mathrm{H}$ and $\mathrm{CO}_{2}$, at the thermodynamic equilibrium, there is a steep variation in composition (smoothed by vertical mixing) corresponding to the strong temperature gradient of the upper atmosphere.

Vertical quenching has an effect on a larger part of the atmosphere of HD 189733b. Both $\mathrm{NH}_{3}$ and $\mathrm{HCN}$ are quenched at 5 bar, $\mathrm{CH}_{4}$ at 1 bar, $\mathrm{H}$ at 40 mbar, and $\mathrm{CO}_{2}$ at 20 mbar. Quenching contaminates the composition up to very lowpressure levels $\left(10^{-4}\right.$ mbar $)$.

\subsection{Comparison with Moses et al. (2011)}

\subsubsection{Equilibrium}

Overall, the composition we calculate at thermodynamic equilibrium (which is our initial condition) is very close to what is obtained by M11 except for HCN for which there is a difference 

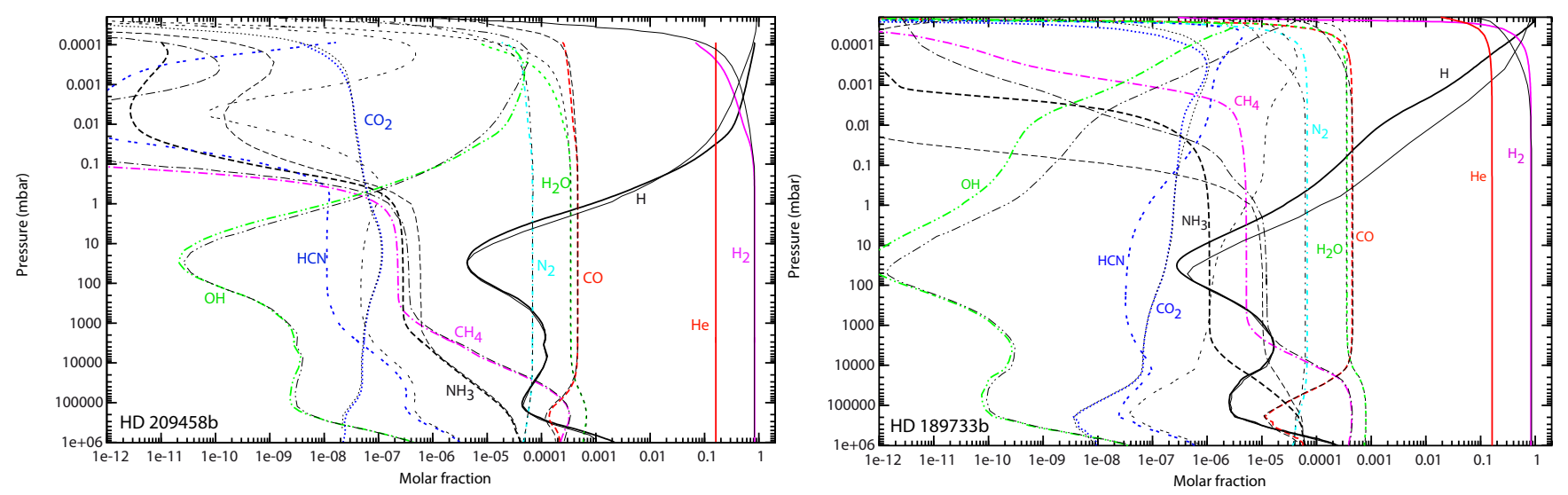

Fig. 7. Comparison between the abundance profiles found by our nominal model (color lines) and by Moses et al. (2011) (thin black lines), for the two planets (HD 209458b (left) and HD 189733b (right)).

that can reach $\sim 30 \%$ at 100 bars and $1545 \mathrm{~K}$. To check our calculations we also did a comparison with the code STANJAN $^{5}$ and found negligible discrepancies for the species we compared, including $\mathrm{HCN}$ at this pressure and temperature. The difference with M11 probably comes from the coefficients used for the NASA polynomials (see Appendix A). Although the difference remains small for equilibrium calculations, we should keep in mind that it may significantly affect the kinetics of $\mathrm{HCN}$ and related species through the calculation of the rate for backward reactions and vertical quenching.

\subsubsection{Steady state}

In Fig. 7, we compare our results at steady state with those of M11. Differences between M11 and our nominal model are also shown species by species in Figs. 8 and 9. Discrepancies between the two models can be due to the different chemical schemes and, at levels where the results are sensitive to photochemistry, to possible differences in the UV fluxes, cross sections, and photodissociation quantum yields. An influence of the numerical implementation (such as the discretization of the atmosphere, the solver for the continuity equations, or the treatment of the UV transfer) is also possible.

In the lower atmosphere of HD 189733b and for most of the atmosphere of HD 209458b, photolyses have a negligible influence and departures should be caused by the kinetics. For these regions we find very similar results for species that remain at their equilibrium abundance $\left(\mathrm{H}, \mathrm{OH}, \mathrm{CO}, \mathrm{CO}_{2}, \mathrm{H}_{2} \mathrm{O}\right.$, for instance), which only confirms, as stated before, that our thermodynamic equilibrium codes are in good agreement. For species quenched by mixing, however, significant deviations appear, in particular for $\mathrm{NH}_{3}, \mathrm{HCN}$, and $\mathrm{CH}_{4}$. Their quenching occurs at different pressure levels and, thus, for different abundances that will then contaminate a large fraction of the atmosphere above. The discrepancies are much more significant in the case of HD $189733 \mathrm{~b}$, due to higher sensitivity to kinetics. Although the kinetic network is certainly the main reason for these departures, it is also true that quenching can be quite sensitive to the resolution of the pressure (or altitude) grid, in particular when there is a steep gradient of temperature which is the case in the convective zone $(P>100$ bar). For this reason, we impose the thickness of individual layers to be smaller than $1 / 8$ th of the local scale height, which results in $~ 300$ layers for the pressure range that we model. Although we do not

\footnotetext{
${ }^{5}$ http://navier.engr.colostate.edu/tools/equil.html
}

know what resolution is used in M11, it seems more likely that the deviation comes from differences in the kinetic network itself. As explained in the Introduction, we use a chemical scheme validated for the species represented and for most of the range of temperature and pressure of the modeled atmospheres. M11, on the other hand, use a chemical scheme derived from Jupiter and Saturn models (Gladstone et al. 1996; Moses 1996; Moses et al. 1995b,a, 2000a,b) completed by high-temperature kinetics from combustion-chemistry literature (Baulch et al. 1992, 1994, 2005; Atkinson et al. 1997, 2006; Smith et al. 1999; Tsang 1987, 1991; Dean \& Bozzelli 2000), which has not, to our knowledge, been validated against experiments. We also note departures in the upper atmosphere, where photolyses are important. In particular, $\mathrm{H}$ and $\mathrm{OH}$ exhibit similar profiles than those of M11 in HD 189733b, but shifted by about one order of magnitude in abundance for pressures lower than 50 mbar. $\mathrm{CH}_{4}$ is also affected. We checked that these differences are not due to using different stellar fluxes by switching between the flux we use and the one used in M11 (for HD 209458b, we both use the solar UV flux). At these altitudes, we note significant sensitivity of the mixing ratio of these species to the Rayleigh scattering, so the treatment of the scattering could explain at least part of this disagreement. Again, and although we do not know the details of the photochemical data and radiative transfer used in M11, we assume that kinetics explain the differences.

\subsection{Other networks for nitrogen species}

The main differences between M11 and our results are related to the quenching of $\mathrm{NH}_{3}$ and $\mathrm{HCN}$. As mentioned in M11, the chemistry of nitrogen compounds has been less studied than carbon species and chemical networks have been subjected to less validation. However, $\mathrm{NO}_{x}, \mathrm{HCN}, \mathrm{CN}$, and $\mathrm{NH}_{3}$ are important species in applied combustion (gas fuel, for instance, can contain high concentrations of ammonia), and should be reproduced well within the temperature and pressure range of the validation. Quenching is found to occur within 1 to 10 mbars, corresponding to the range of validation in terms of pressure. An originality of our network compared to other schemes used in combustion is that it is not optimized to increase the agreement between modeling and experiments. In other words, the rate coefficients of the individual processes were not altered compared to their original measurement or estimate. The application of the network is therefore not strictly restricted to the validation domain.

Other submechanisms are available to model the kinetics of nitrogen-bearing species. They were constructed based 
O. Venot et al.: A chemical model for the atmosphere of hot Jupiters
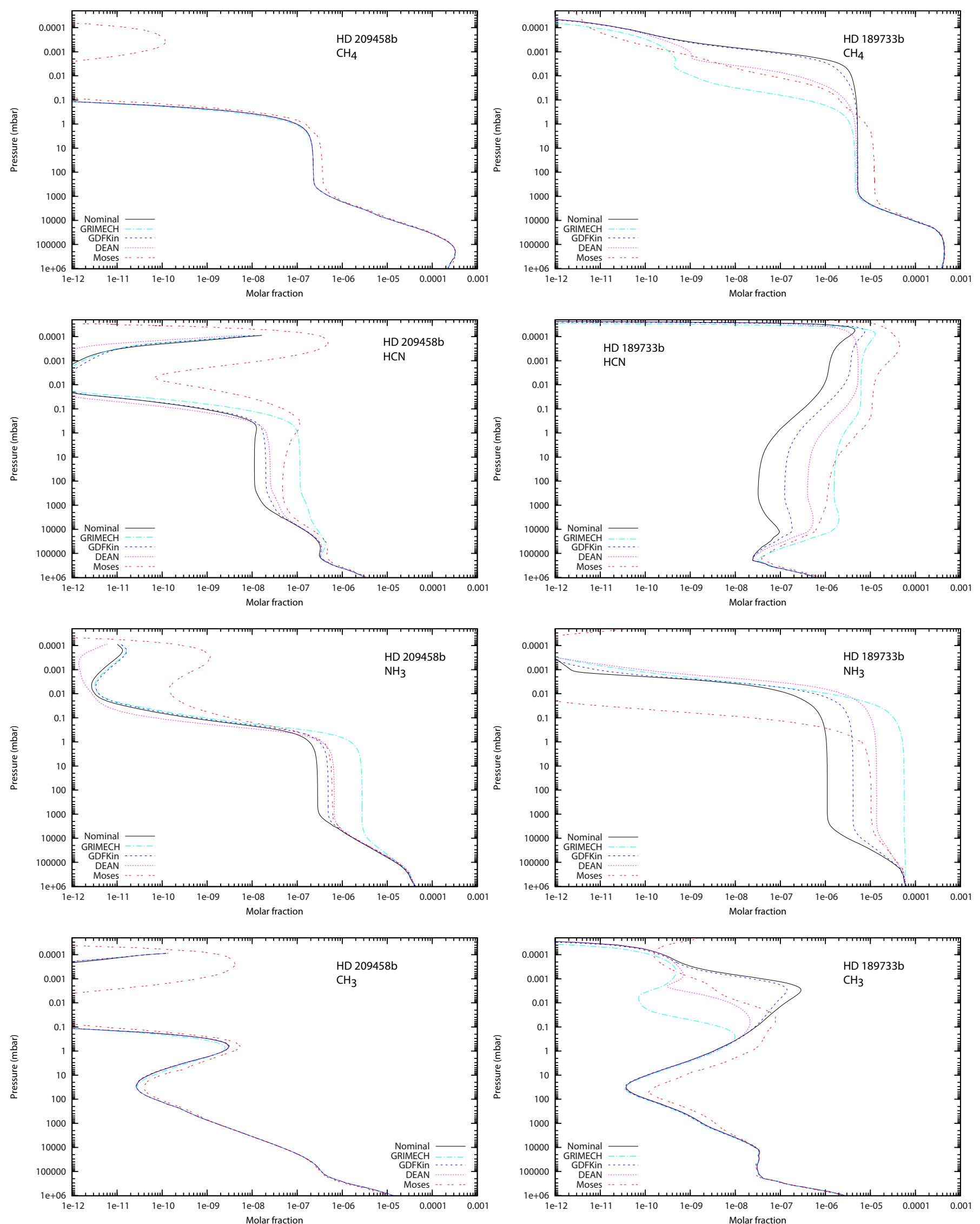

Fig. 8. Abundances of $\mathrm{CH}_{4}, \mathrm{HCN}, \mathrm{NH}_{3}$, and $\mathrm{CH}_{3}$ in $\mathrm{HD} 209458 \mathrm{~b}$ (left) and $\mathrm{HD} 189733 \mathrm{~b}$ (right) with the four different models, compared to the results of Moses et al. (2011). 
A\&A 546, A43 (2012)
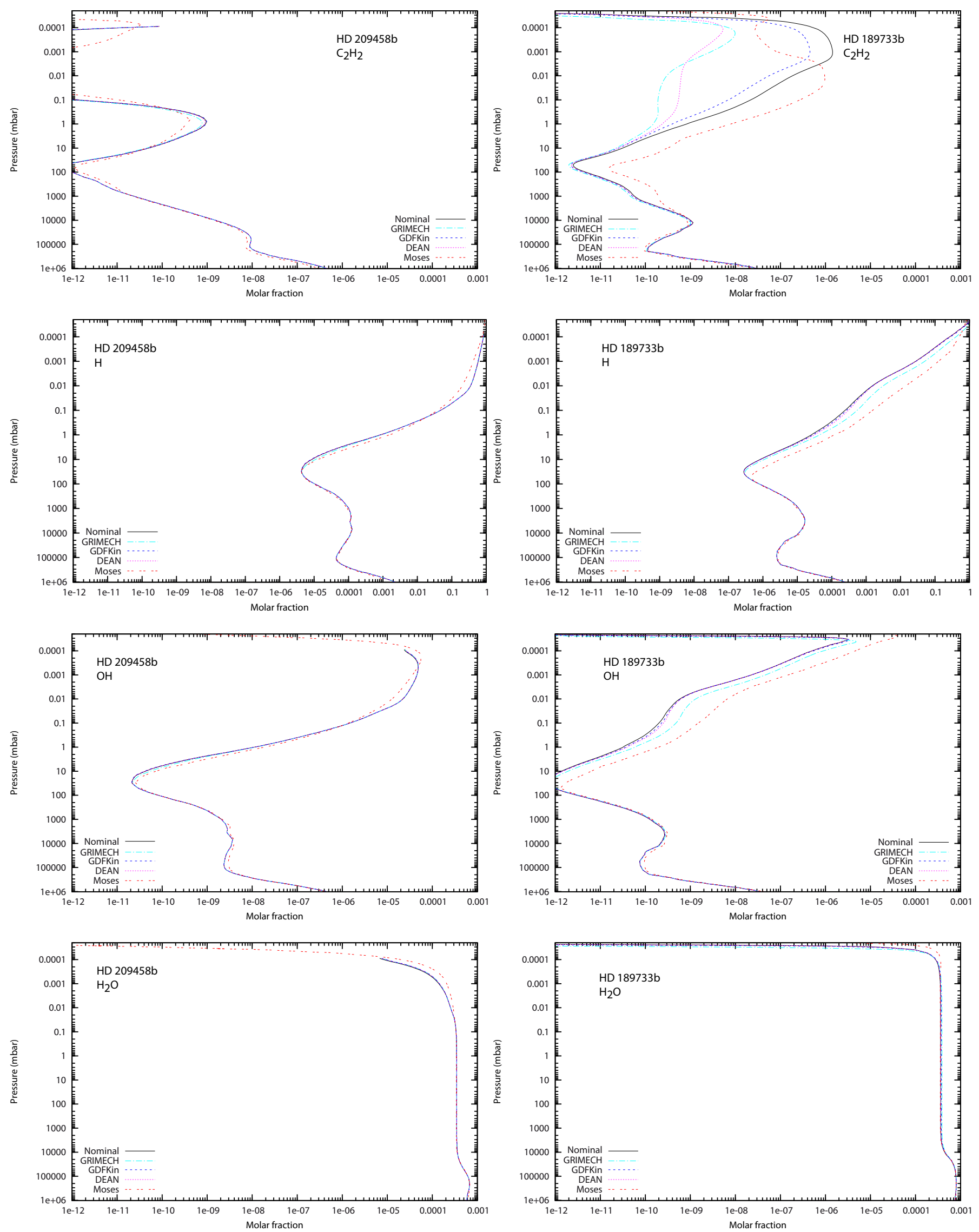

Fig. 9. Abundances of $\mathrm{C}_{2} \mathrm{H}_{2}, \mathrm{H}, \mathrm{OH}$, and $\mathrm{H}_{2} \mathrm{O}$ in $\mathrm{HD} 209458 \mathrm{~b}$ (left) and $\mathrm{HD} 189733 \mathrm{~b}$ (right) with the four different models, compared to the results of Moses et al. (2011). 
on different approaches (optimization, specific domain of application, reduced number of reactions). To test our model against other nitrogen schemes, we replaced our nitrogen reaction base by nitrogen submechanisms taken from other $\mathrm{C} / \mathrm{H} / \mathrm{O} / \mathrm{N}$ mechanisms:

- GRIMECH, mechanism based on GRI-Mech 3.0 (Smith et al. 1999) with several reactions involving $\mathrm{NO}_{x}$ compounds added with respect to the mechanisms of Glaude et al. (2005), as recommended and done by Anderlohr et al. (2009). It includes 162 reversible reactions involving 26 nitrogen compounds. The GRI-Mech 3.0 is a mechanism designed to model natural gas combustion, including NO formation and reburn chemistry. As already mentioned in Sect. 2.1.2, it has been optimized as a global mechanism; i.e., some rate coefficients have been modified (compared to the literature) in order to fit the results of a pool of experiments with conditions and compositions specific to combustion. The individual processes were not studied separately in all the pressure and temperature range. Applying this mechanism beyond its domain of optimization/validation is a risky extrapolation. Mixing ratios of oxidants, for instance, are very low in hot Jupiter atmospheres compared with the experiments used to optimize/validate GRI-Mech 3.0.

While doing the present study, we noticed that two reactions from the GRIMECH mechanism had wrong rates so we corrected them. These erroneous rate constants, which can be traced back to the NIST Chemical Kinetics Database (Manion et al. 2008), were identified by systematically comparing reaction rate constants with collision limit values and energy barriers with the enthalpy budget of the reaction.

The first reaction is

$\mathrm{N}_{2}+\mathrm{H} \longrightarrow \mathrm{N}\left({ }^{4} \mathrm{~S}\right)+\mathrm{N}\left({ }^{4} \mathrm{~S}\right)+\mathrm{H}$

for which the rate given by NIST is $k_{\mathrm{f}}(T)=1.26 \times$ $10^{-9} T^{-0.20} \mathrm{e}^{-27,254 / T} \mathrm{~cm}^{3}$ molecule $\mathrm{s}^{-1}$ (with $T$ in Kelvin), although this expression corresponds in fact to the reaction 7, the thermal dissociation of $\mathrm{NH}$ through collisions with atomic nitrogen (Caridade et al. 2005):

$\mathrm{NH}+\mathrm{N} \longrightarrow \mathrm{H}+\mathrm{N}_{2}$.

The above expression overestimates by many orders of magnitude the rate constant of the reaction, whose activation energy must be around $100000 \mathrm{~K}$, as implied by the bond energy of molecular nitrogen and by measurements of the thermal dissociation of $\mathrm{N}_{2}$ through collisions with various bodies.

We finally adopted a more general form for the dissociation of $\mathrm{N}_{2}$,

$\mathrm{N}_{2}+\mathrm{M} \longrightarrow \mathrm{N}\left({ }^{4} \mathrm{~S}\right)+\mathrm{N}\left({ }^{4} \mathrm{~S}\right)+\mathrm{M}$

with the reaction rate constant $k_{\mathrm{f}}(T)=1.661 \times$ $10^{4} T^{-3.30} \mathrm{e}^{-11,310 / T} \mathrm{~cm}^{3}$ molecule $\mathrm{s}^{-1} \mathrm{~s}^{-1}$ (Thielen \& Roth 1986). This rate had a strong influence on our results. Using the wrong rate has a strong effect on the atmospheric profiles of $\mathrm{NH}_{3}$ and $\mathrm{HCN}$.

The second reaction is

$\mathrm{HONO}+\mathrm{NO} \longrightarrow \mathrm{NO}_{2}+\mathrm{HNO}$

for which the reaction rate constant given by NIST $\left(k_{\mathrm{f}}(T)=\right.$ $7.34 \times 10^{-20} T^{2.64} \mathrm{e}^{-2034 / T} \mathrm{~cm}^{3}$ molecule $\left.{ }^{-1} \mathrm{~s}^{-1}\right)$ was in fact the rate of the reverse of reaction (9), as calculated by Mebel et al. (1998). In our model, we use instead the reaction

$\mathrm{NO}_{2}+\mathrm{HNO} \longrightarrow \mathrm{HONO}+\mathrm{NO}$

with the reaction rate constant $k_{\mathrm{f}}(T)=1.00 \times 10^{-12} \mathrm{e}^{-1000 / T}$ $\mathrm{cm}^{3}$ molecule ${ }^{-1} \mathrm{~s}^{-1}$ (Tsang \& Herron 1991).

When this paper is published, these rates should be corrected in the NIST Chemical Kinetics Database, but one should check that the wrong rates are not used in modeling.

- GDF-Kin, a mechanism optimized for natural gas combustion modeling (Turbiez et al. 1998; De Ferrieres et al. 2008) that includes less individual processes: 180 reversibles reactions involving 22 nitrogen species. Several experimental data on natural gas combustion have been acquired in partnership with Gaz de France to develop this mechanism. The $\mathrm{NO}_{x}$ chemistry has been included in GDF-Kin 3.0 (El Bakali et al. 2006). We used the update version GDF-Kin 5.0 (Lamoureux et al. 2010), in which five reactions involving NCN were refined in order to better reproduce the kinetics of this species. It is validated for temperatures between 400 and $2200 \mathrm{~K}$ and pressures between 0.04 and 10 bars.

- DEAN, taken from Dean \& Bozzelli (2000). This book that presents a catalog of reactions is used by Moses et al. (2011), at least for some reactions. The mechanism derived from this work includes 370 reversible reactions involving 49 nitrogen species and one $\mathrm{C} / \mathrm{H} / \mathrm{O}$ species that is not included in our $\mathrm{C}_{0}-\mathrm{C}_{2}$ scheme: $\mathrm{HCOH}$. The purpose of the work of Dean and Bozzelli was to list gas phase reactions involving nitrogen-bearing species that could be important for hightemperature combustion modeling and to provide the associated rate coefficients based on an analysis of elementary reaction data, when available, or on estimations from thermochemical kinetics principles otherwise. This mechanism was developed on the basis of analysis of individual reactions rather than by attempting to reproduce any specific set of experiments. It is clearly written in this book that "Although we show in the chapter that this mechanism provides a reasonable description of some aspects of high-temperature nitrogen chemistry, we have not attempted a comprehensive comparison". Therefore, this kinetic network should be viewed as a database of reaction rate constants rather than a validated mechanism, in the absence of validation.

The impact of the different nitrogen submechanisms on the abundance profiles of various species is illustrated in Figs. 8 and 9. Thermodynamic equilibrium is the same for all schemes (for the species in common).

First, we restrict our analysis to pressures higher than $\sim 1$ mbar in order not to mix effects of kinetic rates with possible differences in the photochemical data or modeling. For HD 209458b, the main species that are significantly affected at these pressure levels by the change of nitrogen scheme are $\mathrm{HCN}$ and $\mathrm{NH}_{3}$. This is not surprising since these are the most abundant nitrogen species departing from equilibrium due to quenching, and the pressure level at which the quenching occurs depends on the kinetic network adopted. These two species (but also others) show even larger differences in the case of HD 189733b, since the lower temperatures of this atmosphere enhance the differences due to the kinetics. Departures between schemes are expected to become even larger for cooler atmospheres. None of the tested schemes shows an general improvement of the agreement with M11. Similar $\mathrm{NH}_{3}$ quenching is found by M11 and with $D E A N$ for both planets, which makes sense because M11 use Dean \& Bozzelli (2000) as a source of 

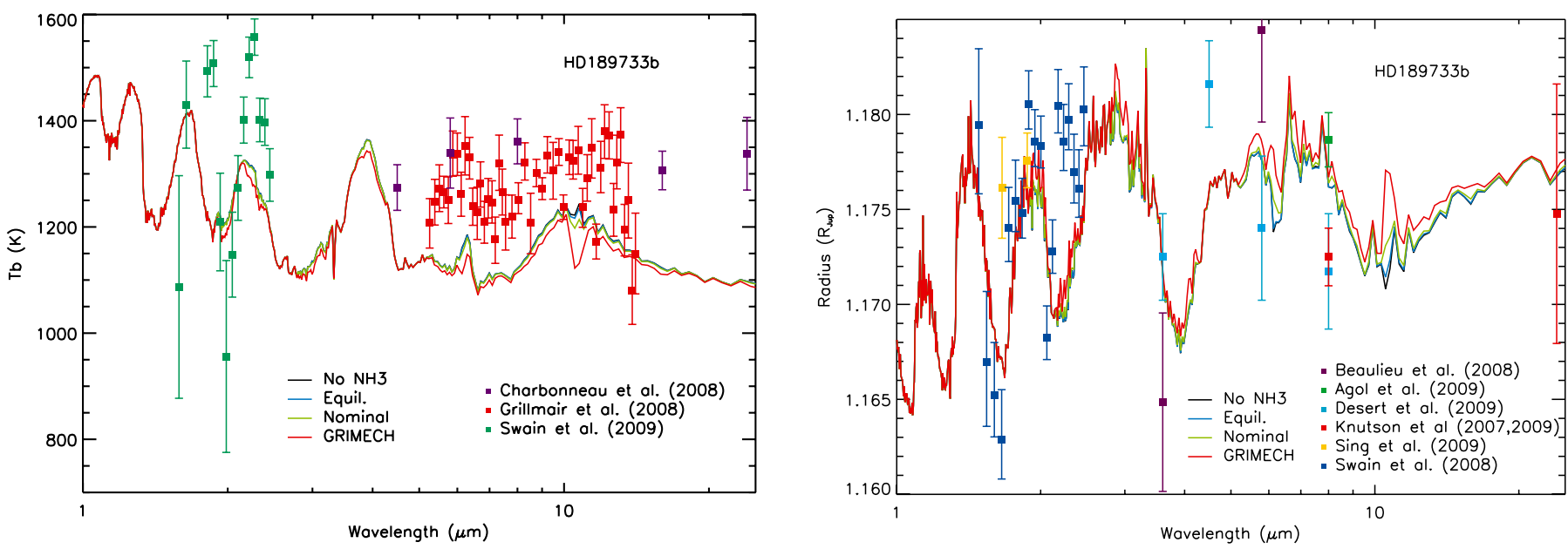

Fig. 10. Synthetic day-side (left) and transmission (right) spectra of HD 189733b with the nominal mechanism (green curve) compared to the one corresponding to the GRIMECH mechanism (red curve) and to the thermochemical equilibrium (blue curve). The dark curve is obtained when $\mathrm{NH}_{3}$ is removed from the model. The day-side fluxes are given as brightness temperatures $\left(T_{\mathrm{b}}\right)$. Because of the reflection component, note that the link between $T_{\mathrm{b}}$ and the atmospheric thermal profile is altered below $2 \mu \mathrm{m}$. The transmission spectrum is given as the apparent planetary radius. The data points obtained from various observations are also shown.

reactions and associated rates for N-bearing compounds. This similarity is also found for HCN but only at pressures higher than 1 bar. At higher altitudes, the HCN profiles from M11 become closer to the result with GRIMECH. For both planets and both $\mathrm{HCN}$ and $\mathrm{NH}_{3}$, profiles obtained with $G D F$-Kin are bracketed by those from the nominal model and DEAN, while GRIMECH gives significantly higher abundances than all other models in the quenching region. With GRIMECH, we also notice that $\mathrm{NH}_{3}$ becomes the main nitrogen-bearing species from the bottom of the atmosphere up to 0.03 mbar because of vertical mixing, whereas thermodynamics predicts that $\mathrm{N}_{2}$ should be the main nitrogen-bearing species.

Understanding the roots of theses discrepancies would require an in-depth study of the sensitivities of these schemes to reaction cycles, as a function of temperature and pressure, which is far beyond the scope of this study and would require tools that may have to be developed specifically for such large networks. To illustrate this difficulty we tried to identify the reaction that dominates the production rate of $\mathrm{NH}_{3}$ for HD $189733 \mathrm{~b}$, at 100 mbar. We found it to be the same for all mechanisms:

$\mathrm{NH}_{2}+\mathrm{H}_{2} \longrightarrow \mathrm{NH}_{3}+\mathrm{H}$,

whose rate constant is similar in all schemes, and is calculated reversing the reaction:

$\mathrm{NH}_{3}+\mathrm{H} \longrightarrow \mathrm{NH}_{2}+\mathrm{H}_{2}$,

which dominates the destruction of $\mathrm{NH}_{3}$, also in all the schemes. The following rates are found in the different schemes:

- Nominal, Dean, GRIMECH: $9.00 \times 10^{-19} T^{2.4} \mathrm{e}^{-4990 / T} \mathrm{~cm}^{3}$ molecule ${ }^{-1} \mathrm{~s}^{-1}$, derived from Ko et al. (1990).

- GDF-Kin: $1.056 \times 10^{-18} T^{2.39} \mathrm{e}^{-5114 / T} \mathrm{~cm}^{3}$ molecule ${ }^{-1} \mathrm{~s}^{-1}$ from Michael et al. (1986).

We could think that these slight differences are responsible for the different results. However, $\mathrm{NH}_{3}$ does not display the same abundance when using Dean and GRIMECH mechanisms, even though they share the same rate constant. Moreover, nulling the rate constant of this reaction in the nominal scheme does not affect the quenching level of $\mathrm{NH}_{3}$ or its abundance for pressures higher than 10 mbar. We can therefore eliminate this hypothesis. Key reactions are in fact usually those that limit the rate of a cycle and that do not dominate the production or destruction of a given species. Finding those limiting processes in complex networks is a field of research in itself.

Identifying key pathways and their limiting reactions require dedicated algorithms (Lehmann 2004; Grenfell et al. 2006; Dobrijevic et al. 2010b; Stock et al. 2011, 2012) whose adaptation to the large networks we consider will require further work.

For hydrocarbons (see for instance $\mathrm{CH}_{4}, \mathrm{C}_{2} \mathrm{H}_{2}$, and $\mathrm{CH}_{3}$ ), all the models we tested cluster to the same profiles for pressures below 1 mbar. This shows that N-bearing species have little influence on hydrocarbon chemistry at these altitudes. (This would no longer be true at higher temperature and for higher $\mathrm{C} / \mathrm{O}$ ratios since $\mathrm{HCN}$ would become a major reservoir of both $\mathrm{N}$ and $\mathrm{C}$.) M11 systematically finds higher mixing ratios for hydrocarbons (but within one order of magnitude) above the quenching level of $\mathrm{CH}_{4}$ (1-10 bar), probably due to kinetic differences in the $\mathrm{C}_{0}-\mathrm{C}_{2}$ mechanism.

At lower pressure, Figs. 8 and 9 show large differences that are no longer due to quenching. At pressures lower than $1 \mathrm{mbar}$, the abundances of hydrocarbons depend on the nitrogen network used. It is particularly striking for $\mathrm{C}_{2} \mathrm{H}_{2}$ in $\mathrm{HD} 189733 \mathrm{~b}$, where $D E A N$ and GRIMECH, on the one hand, and the nominal model, $G D F-K i n$ and M11, on the other hand, seem to cluster in two different regimes, exhibiting two to three orders of magnitude differences at 0.1-0.001 mbar. Departures between network results can come from differences in the kinetic network (different reactions, different rates, different minor species included) but also in photochemistry. Indeed, some UV-absorbing species are not included in all the models, such as $\mathrm{N}_{2} \mathrm{H}_{4}, \mathrm{HNO}_{3}, \mathrm{C}_{2} \mathrm{~N}_{2}$, and $\mathrm{N}_{2} \mathrm{O}_{4}$, which have absorption domains that overlap that of $\mathrm{C}_{2} \mathrm{H}_{2}$.

\subsubsection{Corresponding emission and transmission spectra}

To calculate the planetary transmission and emission+reflection spectra of HD 189733b (Fig. 10) and HD 209458b, we used a line-by-line radiative transfer model from 0.3 to $25 \mu \mathrm{m}$ (Iro et al. 2005; Iro \& Deming 2010). The opacity sources included in the model are the main molecular constituents: $\mathrm{H}_{2} \mathrm{O}, \mathrm{CO}$, $\mathrm{CH}_{4}, \mathrm{NH}_{3}, \mathrm{Na}, \mathrm{K}$, and TiO; collision-induced absorption by $\mathrm{H}_{2}$ 
and $\mathrm{He}$; Rayleigh diffusion; and $\mathrm{H}^{-}$bound-free and $\mathrm{H}_{2}^{-}$freefree. For absorbing species not included in our kinetic model ( $\mathrm{Na}, \mathrm{K}$, and $\mathrm{TiO}$ ), chemical equilibrium is assumed. The current model does not account for clouds. For the reflected component, we used synthetic stellar spectra generated from ATLAS ${ }^{6}$. The main difference from the static model described in Iro et al. (2005) is the addition of $\mathrm{NH}_{3}$ for which we used the HITRAN 2008 database (Rothman et al. 2009). Planetary parameters are taken from Table 1.

We applied this model for the compositions obtained with the two nitrogen mechanisms, which give the most opposite results (Nominal and GRIMECH), as well as for chemical equilibrium. The GRIMECH scheme gives the highest abundance for ammonia: ten times more $\mathrm{NH}_{3}$ than the nominal model for HD 209458b and one hundred times more than the nominal model for HD 189733b. As a consequence, features of this molecule become noticeable on both the emission and transmission spectra at $1.9,2.3,3.0,4.0,6.1$, and $10.5 \mu \mathrm{m}$. The most prominent feature is found for HD $189733 \mathrm{~b}$ at $10.5 \mu \mathrm{m} . \mathrm{NH}_{3}$ features are also visible on the spectra of HD 209458b, but so slightly that it would not be observable.

At the moment, our radiative transfer model does not include the contribution of HCN to the opacities. Based on the HCN abundances and associated spectra found by M11, we can expect the spectra to be also sensitive to the HCN abundance. Indeed, at the altitudes probed by the observations, there is nearly two orders of magnitude less $\mathrm{HCN}$ with our nominal model than with GRIMECH, and GRIMECH gives HCN abundances similar to that of M11. Therefore, the signature of $\mathrm{HCN}$ found by M11 at $14 \mu \mathrm{m}$ should also become noticeable with the GRIMECH version of our scheme.

Some observational data are superimposed on the spectra (Charbonneau et al. 2008; Grillmair et al. 2008; Swain et al. 2009b, for emission spectrum) and (Knutson et al. 2007, 2009; Swain et al. 2008b; Beaulieu et al. 2008; Sing et al. 2009; Désert et al. 2009; Agol et al. 2010, for transmission spectrum), but we do not discuss the agreement between observations and synthetic spectra, as we did not attempt to fit the observable using different thermal profiles.

\section{Discussion}

To study HD 209458b and HD 189733b, we used the pressuretemperature and eddy diffusion profiles from M11. These profiles were derived from general circulation models of Showman et al. (2009). This choice is motivated by the comparison with M11 and also because the actual physical structure of these atmospheres is not yet well constrained by observations; however, Huitson et al. (2012) have recently published thermal profiles for both planets, as inferred from transit spectroscopy. It seems that HD 189733 b could be warmer than HD 209458 b between $10^{-5}$ and $10^{-3}$ bar. Considering the large uncertainties affecting their physical conditions, our models should not be considered as predictions of the composition of hot Jupiters but more as a step in developing chemical models of these objects and model intercomparison.

In addition, circulation is not included in either our model or, to our knowledge, in other current photochemical models of hot Jupiters, although it has a significant influence on the chemical composition of the atmospheres owing, for instance, to the strong longitude dependency of the temperature. We will

\footnotetext{
${ }^{6}$ http://kurucz.harvard.edu/stars.html
}

study the influence of the horizontal transport on the composition of the atmosphere of HD 209458b in a forthcoming paper (Agúndez et al. 2012).

Because modeled abundances depend significantly on the reaction network (in particular for $\mathrm{NH}_{3}, \mathrm{HCN}$, and some hydrocarbons), we recommend using a network that has been validated (but not optimized) against experiments for conditions as close as possible to those of application. We do not claim that the network we release is a definitive one, but it will necessarily evolve as new experimental results and kinetic/thermodynamic data become available. Detailed nitrogen chemistry, for instance, has been implemented in combustion networks only recently and will be subject to further evolution. Missing elements should also be added to the network, sulfur being the most obvious one. Since the scheme has not been optimized, adding new species and reactions to the network we release is possible. Moreover, although the range of $P, T$, and the elements considered in combustion model do fit well with the study of hot Jupiter atmospheres, the ratio between hydrogen and the other elements does not. For this reason, among others, it is important to avoid using optimized networks that would prevent modeling of such hydrogen-rich mixtures. Also, our current network, which cannot be used to study the abundance of species with more than two carbon atoms, is probably insufficient to study atmospheres with $\mathrm{C} / \mathrm{O}$ ratios close to or above unity. For this reason we are currently working on an extended network that can model species up to six carbon atoms.

Hot Jupiter atmospheres represent an extreme case of planetary atmospheres in terms of both high temperatures and low metallicity. With the progress of observations, cooler and heavier atmospheres are or will be soon (with JWST, EChO, Finesse, E-ELT) accessible to characterization. Cooler atmospheres will depart more from equilibrium and will thus be more sensitive to the details of the kinetics. Molecules that remain minor constituents of hot Jupiter atmospheres will become more abundant in cooler atmospheres and have an increased influence on their spectral appearance and thermal structure, making the use of validated schemes even more relevant. More metallic atmospheres should be found as we explore the exoplanet realm towards smaller objects with higher core/envelope masses, and eventually terrestrial objects. While some uncertainties still exist when applying our network to hydrogen-rich atmospheres, atmospheres with a decreasing hydrogen-to-heavy elements ratio become closer to the conditions of validation (by their equivalence ratios, to use combustion terms). Even hot Jupiters can be significantly enriched (by factor of 10 or more) in heavy elements compared to their parent stars, which could, for instance, explain the high observed abundances of $\mathrm{CO}_{2}$ (Zahnle et al. 2009a).

\section{Conclusion}

We have constructed a chemical scheme to study the atmospheric composition of hot Jupiters. Compared to existing models we chose to use one that

- is derived from mechanisms that are intensively used for industrial applications (in particular car engine simulations);

- has been subjected to in-depth validation protocols in a broad range of temperatures, pressures and compositions;

- is based on individual rate coefficients that have not been altered to optimize the agreement between the collective behavior of the network and experiments (contrary to most mechanisms in combustion). This allows users to apply the 
network slightly beyond its validation domain and to add species and reactions;

- uses experimental measurements for some of the endothermic reactions when robust data are available but still reproduces thermodynamic equilibrium with excellent accuracy.

We developed a 1D photochemical model based on this kinetic scheme, which includes vertical transport (mixing and molecular diffusion) and photodissociations. We applied this model to the hot Jupiters HD 209458b and HD 189733b and compared our results with those of Moses et al. (2011). Qualitatively, we find similar conclusions: photodissociations do not have a significant impact on the atmospheric composition of HD 209458b, with the high temperatures we assume. It remains at the thermodynamic equilibrium for pressures higher than 1 bar. For lower pressures, vertical transport affects the abundances of $\mathrm{HCN}, \mathrm{NH}_{3}, \mathrm{CH}_{4}$, and some of the minor associated species. For HD 189733b, we assume significantly lower temperatures and find the atmospheric composition to be more sensitive to photolyses and vertical transport, with all species affected, except the main reservoirs, $\mathrm{H}_{2}, \mathrm{H}_{2} \mathrm{O}, \mathrm{CO}$, and $\mathrm{N}_{2}$. Quantitatively, however, we find significant differences (up to several orders of magnitude in the case of HD 189733b) in the abundances that are likely to be due to the different chemical schemes used. These differences are smaller for HD 209548b because kinetics have less influence. The quenching of $\mathrm{HCN}$ and $\mathrm{NH}_{3}$, as well as $\mathrm{CH}_{4}$ to a less extent, is particularly affected, as well as most species sensitive to photochemistry in the upper atmosphere. Despite being large in terms of abundances, these differences do not produce strong effects on the spectra, with the exception of $\mathrm{NH}_{3}$ at $10.5 \mu \mathrm{m}$. Comparing different schemes with observations will thus have to await more accurate spectroscopic observations (JWST, E-ELT, EChO). Until them, experimental validation appears mandatory.

To illustrate the sensitivity to the kinetic scheme, we implemented different available nitrogen schemes that are either optimized (GRIMECH, GDFKin) or not validated (Dean). We studied the extent of the possible results, and found large differences whenever disequilibrium chemistry is at work. Changing the nitrogen scheme strongly affects the quenched species (HCN, $\mathrm{NH}_{3}$ ) and most species (including hydrocarbons) in the upper atmosphere of HD 189733b. For HD 209458b, deviations are again less noticeable since the atmosphere departs less from equilibrium. We therefore emphasize the need to use validated and nonoptimized chemical schemes. This is already true for hot Jupiters but is even more crucial in the case of cooler atmospheres (GJ1614b, GJ3470b, for instance), which depart more from thermodynamic equilibrium and are more sensitive to kinetics.

Our nominal scheme can be downloaded from the KIDA database $^{7}$ (Wakelam et al. 2012). The scheme is designed to reproduce the kinetic evolution of species with fewer than two carbon atoms. To study atmospheres with $\mathrm{C} / \mathrm{O}$ ratios higher than solar (close to or above 1 ), we are currently developing a $\mathrm{C}_{0}-\mathrm{C}_{6}$ scheme that will be able to describe the kinetics of species up to six carbon atoms. One of the next improvements to our model should be the addition of sulfur. Because the kinetics of nitrogen species is an active field of research, we expect regular updates of the network (which would be notified and available on KIDA).

Any conclusions from this study of the chemical composition of hot Jupiters, which derive from models using an average $1 \mathrm{D}$ vertical profile, will probably have to be revisited with the effects of atmospheric circulation.

\footnotetext{
7 http://kida.obs.u-bordeaux1.fr/
}

Acknowledgements. We thank Ignasi Ribas for providing the UV flux of $\epsilon$ Eridani, proxy of HD 189733 and Anthony M. Dean for providing the list of rate coefficients from his book in an electronic form. F.S., O.V., E.H., and M.A. acknowledge support from the European Research Council (ERC Grant 209622: $\mathrm{E}_{3} \mathrm{ARTHs}$ ).

\section{References}

Agol, E., Cowan, N., Knutson, H., et al. 2010, ApJ, 721, 1861 Agúndez, M., Venot, O., Iro, N., et al. 2012, A\&A, submitted Akimoto, H., Obi, K., \& Tanaka, I. 1965, J. Chem. Phys., 42, 3864

Anderlohr, J., Bounaceur, R., Pires Da Cruz, A., \& Battin-Leclerc, F. 2009, Combustion and Flame, 156, 505

Anderlohr, J., Pires da Cruz, A., Bounaceur, R., \& Battin-Leclerc, F. 2010, Combustion Sci. Techn., 182, 39

Atakan, B., \& Hartlieb, A. 2000, Appl. Phys. B: Lasers Opt., 71, 697

Atkinson, R., Baulch, D., Cox, R., et al. 1997, J. Phys. Chem. Ref. Data, 26, 1329

Atkinson, R., Baulch, D., Cox, R., et al. 2006, Atmos. Chem. Phys., 6, 3625

Au, J., Cooper, G., Burton, G., Olney, T., \& Brion, C. 1993, Chem. Phys., 173, 209

Balucani, N., Alagia, M., Cartechini, L., et al. 2000a, J. Am. Chem. Soc., 122, 4443

Balucani, N., Cartechini, L., Alagia, M., Casavecchia, P., \& Volpi, G. 2000b, J. Phys. Chem. A, 104, 5655

Barbé, P., Battin-Leclerc, F., \& Côme, G. 1995, J. Chim. Phys., 92, 1666

Barman, T. 2007, ApJ, 661, L191

Batalha, N. M., Rowe, J. F., Bryson, S. T., et al. 2012, ApJS, submitted [arXiv: 1202.5852]

Battin-Leclerc, F., Bounaceur, R., Belmekki, N., \& Glaude, P. 2006, Int. J. Chem. Kin., 38, 284

Battin-Leclerc, F., Blurock, E., Bounaceur, R., et al. 2011, Chem. Soc. Rev., 40, 4762

Baulch, D., Cobos, C., Cox, R., et al. 1992, J. Phys. Chem. Ref. Data, 21, 411

Baulch, D., Cobos, C., Cox, R., et al. 1994, J. Phys. Chem. Ref. Data, 23, 847

Baulch, D., Bowman, C., Cobos, C., et al. 2005, J. Phys. Chem. Ref. Data, 34, 757

Beaulieu, J., Carey, S., Ribas, I., \& Tinetti, G. 2008, ApJ, 677, 1343

Beaulieu, J., Kipping, D., Batista, V., et al. 2010, MNRAS, 409, 963

Benson, S. W. 1976, Thermochemical Kinetics: methods for the estimation of thermochemical data and rate parameters, second edition (New York: John Wiley)

Benson, S. W., \& Cohen, N. 1997, In Peroxyl Radicals (New York: John Wiley) Bounaceur, R., Glaude, P., Fournet, R., et al. 2007, Int. J. Vehicle Design, 44, 124

Bounaceur, R., Herbinet, O., Fournet, R., et al. 2010, SAE Tech. Paper, 01

Brown, M. J., \& Smith, D. B. 1994, 25th Symposium (International) on Combustion, 25, 1011

Burrows, A., \& Sharp, C. 1999, ApJ, 512, 843

Burrows, A., Hubeny, I., Budaj, J., Knutson, H., \& Charbonneau, D. 2007, ApJ, 668, L171

Burrows, A., Budaj, J., \& Hubeny, I. 2008, ApJ, 678, 1436

Burton, G., Chan, W., Cooper, G., \& Brion, C. 1993, Chem. Phys., 177, 217

Caridade, P., Rodrigues, S., Sousa, F., \& Varandas, A. 2005, J. Phys. Chem. A, 109,2356

Cavalié, T., Billebaud, F., Biver, N., et al. 2008, Planet. Space Sci., 56, 1573

Cavalié, T., Billebaud, F., Dobrijevic, M., et al. 2009, Icarus, 203, 531

Chan, W., Cooper, G., \& Brion, C. 1992, Chem. Phys., 168, 375

Chan, W., Cooper, G., \& Brion, C. 1993a, Chem. Phys., 170, 111

Chan, W., Cooper, G., \& Brion, C. 1993b, Chem. Phys., 178, 387

Chan, W., Cooper, G., Sodhi, R., \& Brion, C. 1993c, Chem. Phys, 170, 81

Chang, A. H. H., Mebel, A. M., Yang, X.-M., Lin, S. H., \& Lee, Y. T. 1998, J.

Chem. Phys., 109, 2748

Charbonneau, D., Brown, T., Latham, D., \& Mayor, M. 2000, ApJ, 529, L45

Charbonneau, D., Brown, T., Noyes, R., \& Gilliland, R. 2002, ApJ, 568, 377

Charbonneau, D., Knutson, H., Barman, T., et al. 2008, ApJ, 686, 1341

Chen, F., \& Wu, C. 2004, J. Quant. Spectr. Rad. Trans., 85, 195

Chen, F., Judge, D., Wu, C., \& Caldwell, J. 1999, Planet. Space Sci., 47, 261

Cheng, B., Lu, H., Chen, H., et al. 2006, ApJ, 647, 1535

Cody, R., Sabetydzvonik, M., \& Jackson, W. 1977, J. Chem. Phys., 66, 2145

Cooper, C., \& Showman, A. 2006, ApJ, 649, 1048

Cooper, G., Burton, G., \& Brion, C. 1995a, J. Electron Spectros. Rel. Phen., 73, 139

Cooper, G., Olney, T., \& Brion, C. 1995b, Chem. Phys., 194, 175

Cooper, G., Anderson, J., \& Brion, C. 1996, Chem. Phys., 209, 61 
Coppens, F., De Ruyck, J., \& Konnov, A. 2007, Combustion and Flame, 149, 409

Dagaut, P., Glarborg, P., \& Alzueta, M. 2008, Progress in Energy and Combustion Science, 34, 1

De Ferrieres, S., El Bakali, A., Lefort, B., Montero, M., \& Pauwels, J. 2008, Combustion and Flame, 154, 601

Dean, A., \& Bozzelli, J. 2000, Springer-Verlag Inc, 125

Désert, J., Lecavelier des Etangs, A., Hébrard, G., et al. 2009, ApJ, 699, 478

Dirrenberger, P., Le Gall, H., Bounaceur, R., et al. 2011, Energy \& Fuels, 15, 3875

Dobrijevic, M., Ollivier, J., Billebaud, F., Brillet, J., \& Parisot, J. 2003, A\&A, 398, 335

Dobrijevic, M., Cavalié, T., Hébrard, E., et al. 2010a, Planet. Space Sci., 1555

Dobrijevic, M., Hébrard, E., Plessis, S., et al. 2010b, Adv. Space Res., 45, 77

Domalski, E. S., \& Hearing, E. D. 1996, J. Phys. Chem. Ref. Data, 25, 1

El Bakali, A., Pillier, L., Desgroux, P., et al. 2006, Fuel, 85, 896

Eng, R., Lambert, H., Fei, R., Carrington, T., \& Filseth, S. 1996, Chem. Phys. Lett., 261, 651

Eriksson, G., \& Rosen, E. 1971, Acta Polytechnica Scandinavica - Chemical Including Metallurgy Series, 2

Fahr, A., Hassanzadeh, P., \& Atkinson, D. 1998, Chem. Phys., 236, 43

Fillion, J., Ruiz, J., Yang, X., et al. 2004, J. Chem. Phys., 120, 6531

Fortney, J., Cooper, C., Showman, A., Marley, M., \& Freedman, R. 2006, ApJ, 652,746

Fortney, J., Lodders, K., Marley, M., \& Freedman, R. 2008a, ApJ, 678, 1419

Fortney, J., Marley, M., Saumon, D., \& Lodders, K. 2008b, ApJ, 683, 1104

Fournet, R., Baugé, J., \& Battin-Leclerc, F. 1999, Int. J. Chem. Kin., 31, 361

Gans, B., Boye-Peronne, S., Broquier, M., et al. 2011, Phys. Chem. Chem. Phys., 13,8140

Gardner, J. P., Mather, J. C., Clampin, M., et al. 2006, Space Sci. Rev., 123, 485

Gibbs, J. 1873, A method of geometrical representation of the thermodynamic properties of substances by means of surfaces (Connecticut Academy of Arts and Sciences)

Gladstone, G., Allen, M., \& Yung, Y. 1996, Icarus, 119, 1

Glaude, P., Marinov, N., Koshiishi, Y., Matsunaga, N., \& Hori, M. 2005, Energy \& Fuels, 19, 1839

Golden, D. 2008, Int. J. Chem. Kin., 40, 310

Grenfell, J., Lehmann, R., Mieth, P., Langematz, U., \& Steil, B. 2006, J. Geophys. Res., 111, D17311

Grillmair, C., Burrows, A., Charbonneau, D., et al. 2008, Nature, 456, 767

Hampson Jr, R., \& McNesby, J. 1965, J. Chem. Phys., 42, 2200

Hauschildt, P., Allard, F., \& Baron, E. 1999, ApJ, 512, 377

Hébrard, E., Dobrijevic, M., Bénilan, Y., \& Raulin, F. 2006, J. Photochem. Photobiol. C: Photochem. Rev., 7, 211

Hébrard, E., Dobrijevic, M., Bénilan, Y., \& Raulin, F. 2007, Planet. Space Sci., 55,1470

Herron, J. 1999, J. Phys. Chem. Ref. Data, 28, 1453

Hochanadel, C., Sworski, T., \& Ogren, P. 1980, J. Phys. Chem., 84, 231

Holland, D., Shaw, D., Hayes, M., et al. 1997, Chem. Phys., 219, 91

Howard, A., Marcy, G., Johnson, J., et al. 2010, Science, 330, 653

Howard, A., Marcy, G. W., Bryson, S. T., et al. 2012, ApJS, 201, 15

Huebner, W., Keady, J., \& Lyon, S. 1992, Solar photo rates for planetary atmospheres and atmospheric pollutants (Kluwer Academic Pub)

Huestis, D. L., \& Berkowitz, J. 2010, Adv. Geosci., 25, 229

Huffman, R. 1969, Canadian J. Chem., 47, 1823

Huitson, C. M., Sing, D. K., Vidal-Madjar, A., et al. 2012, MNRAS, 422, 2477

Iida, Y., Carnovale, F., Daviel, S., \& Brion, C. 1986, Chem. Phys., 105, 211

Iro, N. \& Deming, L. D. 2010, ApJ, 712, 218

Iro, N., Bézard, B., \& Guillot, T. 2005, A\&A, 436, 719

Isaksen, I. S. A., Midtbo, K. H., Sunde, J., \& Crutzen, P. J. 1977, Geophys. Norvegica, 31, 11

Ityakov, D., Linnartz, H., \& Ubachs, W. 2008, Chem. Phys. Lett., 462, 31

Jackson, W. \& Halpern, J. 1979, J. Chem. Phys., 70, 2373

Kameta, K., Machida, S., Kitajima, M., et al. 1996, J. Electron Spectroscopy and Related Phenomena, 79, 391, Proceedings of the 11th International Conference on Vacuum Ultraviolet Radiation Physics

Kameta, K., Kouchi, N., Ukai, M., \& Hatano, Y. 2002, J. Electr. Spectr. Rel. Phenom., 123, 225

Khamaganov, V., Karunanandan, R., Rodriguez, A., \& Crowley, J. 2007, Phys. Chem. Chem. Phys., 9, 4098

Knutson, H. A., Charbonneau, D., Allen, L. E., et al. 2007, Nature, 447, 183

Knutson, H., Charbonneau, D., Cowan, N., et al. 2009, ApJ, 690, 822

Ko, T., Marshall, P., \& Fontijn, A. 1990, J. Phys. Chem., 94, 1401

Konnov, A. A. 2000, in 28th Symposium (Int.) on Combustion. Abstr. Symp. Pap, 317

Konnov, A. A. 2009, Combustion and Flame, 156, 2093

Konnov, A. A., \& De Ruyck, J. 1999a, Combustion Sci. Techn., 149, 53
Konnov, A. A., \& De Ruyck, J. 1999b, in Mediterranean Combustion Symposium, 679

Konnov, A. A., \& De Ruyck, J. 2000a, in 16th International Symposium on Gas Kinetics, Cambridge, Abstr. Symp. Pap. PC10

Konnov, A. A., \& De Ruyck, J. 2000b, Combustion Sci. Techn., 152, 23

Konnov, A. A., \& De Ruyck, J. 2001a, Combustion and Flame, 125, 1249

Konnov, A. A., \& De Ruyck, J. 2001b, Combustion and Flame, 124, 106

Lamoureux, N., Desgroux, P., El Bakali, A., \& Pauwels, J. 2010, Combustion and Flame, 157, 1929

Langland-Shula, L., Vogt, S., Charbonneau, D., Butler, P., \& Marcy, G. 2009, ApJ, 696, 1355

Lee, L. 1980, J. Chem. Phys., 72, 6414

Lee, A., Yung, Y., Cheng, B., et al. 2001, ApJ, 551, L93

Lehmann, R. 2004, J. Atmos. Chem., 47, 45

Liang, M., Parkinson, C., Lee, A., Yung, Y., \& Seager, S. 2003, ApJ, 596, L247

Liang, M., Seager, S., Parkinson, C., Lee, A., \& Yung, Y. 2004, ApJ, 605, L61

Lias, S. G., Collin, G. J., Rebbert, R. E., \& Ausloos, P. 1970, J. Chem. Phys., 52, 1841

Lindemann, F., Arrhenius, S., Langmuir, I., et al. 1922, Tran. Faraday Soc., 17, 598

Line, M., Liang, M., \& Yung, Y. 2010, ApJ, 717, 496

Line, M., Vasisht, G., Chen, P., Angerhausen, D., \& Yung, Y. 2011, ApJ, 738,

Lodders, K., \& Fegley, B. 2002, Icarus, 155, 393

Lodders, K., \& Palme, H. 2009, Meteor. Planet. Sci. Suppl., 72, 5154

Loison, J.-C., Kable, S. H., Houston, P. L., \& Burak, I. 1991, J. Chem. Phys., 94, 1796

Luo, Y. 2003, Handbook of bond dissociation energies in organic compounds (CRC)

Madhusudhan, N., \& Seager, S. 2009, ApJ, 707, 24

Madhusudhan, N., \& Seager, S. 2010, ApJ, 725, 261

Madhusudhan, N., Harrington, J., Stevenson, K., et al. 2011a, Nature, 469, 64

Madhusudhan, N., Mousis, O., Johnson, T., \& Lunine, J. 2011b, ApJ, 743, 191

Manion, J. A., Huie, R. E., Levin, R. D., et al. 2008, NIST Chemical Kinetics Database, NIST Standard Reference Database 17

McBride, B., Gordon, S., \& Reno, M. 1993, NASA Technical Memorandum, 4513

McNesby, J., Tanaka, I., \& Okabe, H. 1962, J. Chem. Phys., 36, 605

Mebel, A. M., Lin, M. C., \& Morokuma, K. 1998, Int. J. Chem. Kin., 30

Meller, R., \& Moortgat, G. 2000, J. Geophys. Res., 105, 7089

Merienne, M., Jenouvrier, A., Coquart, B., \& Lux, J. 1997, J. Atmos. Chem., 27, 219

Michael, J., Sutherland, J., \& Klemm, R. 1986, J. Phys. Chem., 90, 497

Moses, J. I. 1996, in The Collision of Comet Shoemaker-Levy 9 and Jupiter, eds. H. A. Weaver, K. S. Noll, P. D. Feldman (Cambridge, UK: Cambridge Univ. Press)

Moses, J., Allen, M., \& Gladstone, G. 1995a, Geophys. Res. Lett., 22, 1601

Moses, J., Allen, M., \& Gladstone, G. 1995b, Geophys. Res. Lett., 22, 1597

Moses, J., Bézard, B., Lellouch, E., et al. 2000a, Icarus, 143, 244

Moses, J., Lellouch, E., Bézard, B., et al. 2000b, Icarus, 145, 166

Moses, J., Visscher, C., Fortney, J., et al. 2011, ApJ, 737, 15

Mota, R., Parafita, R., Giuliani, A., et al. 2005, Chem. Phys. Lett., 416, 152

Mount, G., \& Moos, H. 1978, ApJ, 224, L35

Muller, C., Michel, V., Scacchi, G., \& Côme, G. 1995, J. Chim. Phys., 92, 1154

Okabe, H. 1978, Photochemistry of small molecules (Wiley New York)

Okabe, H. 1981, J. Chem. Phys., 75, 2772

Okabe, H. 1983, J. Chem. Phys., 78, 1312

Olney, T., Cann, N., Cooper, G., \& Brion, C. 1997, Chem. Phys., 223, 59

Orkin, V., Huie, R., \& Kurylo, M. 1997, J. Phys. Chem. A, 101, 9118

Parkes, D., Paul, D., Quinn, C., \& Robson, R. 1973, Chem. Phys. Lett., 23, 425

Reynolds, W. C. 1986, Technical Report (Stanford: Stanford University)

Richardson, L., Deming, D., Horning, K., Seager, S., \& Harrington, J. 2007, Nature, 445, 892

Rothman, L. S., Gordon, I. E., Barbe, A., et al. 2009, J. Quant. Spectr. Rad. Trans., 110, 533

Rowe, J., Matthews, J., Seager, S., et al. 2008, ApJ, 689, 1345

Samson, J., \& Cairns, R. 1964, J. Geophys. Res., 69, 4583

Samson, J., \& Haddad, G. 1994, JOSA B, 11, 277

Sander, S. P., Friedl, R. R., Barker, J. R., et al. 2011, NASA/JPL Publication

Sanz-Forcada, J., Micela, G., Ribas, I., et al. 2011, A\&A, 532, A6

Sato, K., Misawa, K., Kobayashi, Y., et al. 1999, J. Phys. Chem. A, 103, 8650

Schultz, E., \& Shepherd, J. 1999, California Institute of Technology, Graduate Aeronautical Laboratories, Technical Report FM99-5 
Seager, S., \& Sasselov, D. 2000, ApJ, 537, 916

Selsis, F., Despois, D., \& Parisot, J. 2002, A\&A, 388, 985

Sharp, C., \& Burrows, A. 2007, ApJS, 168, 140

Showman, A., Fortney, J., Lian, Y., et al. 2009, ApJ, 699, 564

Sing, D., Vidal-Madjar, A., Désert, J., Etangs, A., \& Ballester, G. 2008, ApJ, 686, 658

Sing, D., Désert, J., des Etangs, A., et al. 2009, A\&A, 505, 891

Smith, G., Golden, D., Frenklach, M., et al. 1999,

URL: http://www.me.berkeley.edu/gri_mech

Smith, W., \& Missen, R. 1982, Chemical reaction equilibrium analysis: theory and algorithms (New York: Wiley)

Southworth, J. 2008, MNRAS, 386, 1644

Southworth, J. 2010, MNRAS, 408, 1689

Stark, G., Smith, P. L., Huber, K. P., et al. 1992, J. Chem. Phys., 97, 4809

Stark, G., Yoshino, K., Smith, P. L., \& Ito, K. 2007, J. Quant. Spectros. Rad. Trans., 103, 67

Stewart, P., Larson, C., \& Golden, D. 1989, Combustion and Flame, 75, 25

Stock, J., Grenfell, J., Lehmann, R., Patzer, A., \& Rauer, H. 2011, Planet. Space Sci., 68, 18

Stock, J., Boxe, C., Lehmann, R., et al. 2012, Icarus, 219, 13

Stockwell, W. \& Calvert, J. 1978, J. Photochem., 8, 193

Swain, M. R. 2010, in BAAS, 42, AAS/Division for Planetary Sciences Meeting Abstracts, 42, 1064

Swain, M., Bouwman, J., Akeson, R., Lawler, S., \& Beichman, C. 2008a, ApJ, 674,482

Swain, M., Vasisht, G., \& Tinetti, G. 2008b, Nature, 452, 329

Swain, M., Tinetti, G., Vasisht, G., et al. 2009a, ApJ, 704, 1616

Swain, M., Vasisht, G., Tinetti, G., et al. 2009b, ApJ, 690, L114

Thielen, K., \& Roth, P. 1986, AIAA journal, 24, 1102

Tinetti, G., Vidal-Madjar, A., Liang, M., et al. 2007, Nature, 448, 169
Tinetti, G., Beaulieu, J. P., Henning, T., et al. 2012, Exper. Astron., in press [arXiv: 1112.2728]

Troe, J. 1983, Berichte der Bunsengesellschaft Phys. Chem., 87, 161

Troe, J. 2011, Combustion and Flame, 158, 594

Tsang, W. 1987, J. Phys. Chem. Ref. Data, 16, 471

Tsang, W. 1991, J. Phys. Chem. Ref. Data, 20, 221

Tsang, W., \& Hampson, R. 1986, J. Phys. Chem. Ref. Data, 15, 1087

Tsang, W., \& Herron, J. 1991, J. Phys. Chem. Ref. Data, 20, 609

Turbiez, A., Pauwels, J., Desgroux, P., et al. 1998, in 27th Symposium (international) on Combustion

Umemoto, H., Nakae, T., Hashimoto, H., \& Kongo, K. 1998, J. Chem. Phys., 109,5844

Vaghjiani, G. L. 1993, J. Chem. Phys., 98, 2123

Vaghjiani, G. 1995, Int. J. Chem. Kin., 27, 777

Van Dishoeck, E., \& Dalgarno, A. 1984, Icarus, 59, 305

Vandaele, A., Hermans, C., Simon, P., et al. 1998, J. Quant. Spectros. Rad. Trans., 59, 171

Visscher, C., Lodders, K., \& Fegley, Jr., B. 2006, ApJ, 648, 1181

Visscher, C., Lodders, K., \& Fegley, Jr., B. 2010, ApJ, 716, 1060

Wakelam, V., Herbst, E., Loison, J.-C., et al. 2012, ApJS, 199, 21

Wang, H., Warner, S., Oehlschlaeger, M., et al. 2010, Combustion and Flame, 157,1976

White, W., Johnson, S., \& Dantzig, G. 1958, J. Chem. Phys., 28, 751

Wu, C., Chen, F., \& Judge, D. 2001, J. Geophys. Res., 106, 7629

Wu, C., Chen, F., \& Judge, D. 2004, J. Geophys. Res., 109, E07S15

Zahnle, K., Marley, M., Freedman, R., Lodders, K., \& Fortney, J. 2009a, ApJ, 701, L20

Zahnle, K., Marley, M. S., \& Fortney, J. J. 2009b, ApJL, submitted [arXiv:0911.0728] 


\section{Appendix A: Thermochemical data}

Thermochemical properties, such as enthalpies of formation, entropies, and heat capacities are very important to ensure the consistency between the rate parameters of the forward and reverse elementary reactions. They are also useful for estimating the heat release rate. Thermochemical data for all molecules or radicals have been estimated and stored as 14 NASA polynomial coefficients, according to the McBride et al. (1993) formalism. The NASA polynomials take the following form:

$$
\begin{aligned}
& \frac{c_{p}^{0}(T)}{R T}=a_{1}+a_{2} T+a_{3} T^{2}+a_{4} T^{3}+a_{5} T^{4} \\
& \frac{h^{0}(T)}{R T}=a_{1}+a_{2} \frac{T}{2}+a_{3} \frac{T^{2}}{3}+a_{4} \frac{T^{3}}{4}+a_{5} \frac{T^{4}}{5}+\frac{a_{6}}{T} \\
& \frac{s^{0}(T)}{R}=a_{1} \ln T+a_{2} T+a_{3} \frac{T^{2}}{2}+a_{4} \frac{T^{3}}{3}+a_{5} \frac{T^{4}}{4}+a_{7},
\end{aligned}
$$

where $a_{i}, i \in[1,7]$, are the numerical NASA coefficients for the fourth-order polynomial. Each species is characterized by fourteen numbers. The first seven numbers are for the high-temperature range, generally from 1000 to $5000 \mathrm{~K}$, and the following seven numbers are the coefficients for the lowtemperature range, generally from 300 to $1000 \mathrm{~K}$. When these parameters are not available in the literature (McBride et al. 1993) or in databases ${ }^{8}$, which is the most frequent case for species present in automotive fuels, they have to be estimated. In this case, these data were automatically calculated using the software THERGAS (Muller et al. 1995), which was developed in the LRGP laboratory and is based on the group and bond additivity methods proposed by Benson (1976) and updated based on the data of Domalski \& Hearing (1996). The enthalpies of formation of alkyl radicals have been also updated according to the values of bond dissociation energies published by Tsang \& Hampson (1986) and by Luo (2003) and following the recommendations of Benson \& Cohen (1997).

An elementary reversible reaction $i$ involving $L$ chemical species can be represented in the general form

$$
\sum_{l=1}^{L} v_{l i}^{\prime} \chi_{l} \Leftrightarrow \sum_{l=1}^{L} v_{l i}^{\prime \prime} \chi_{l}
$$

where $v_{l i}^{\prime}$ are the forward stoichiometric coefficients, and $v_{l i}^{\prime \prime}$ are the reverse ones. $\chi_{l}$ is the chemical symbol of the $l$ th species.

The kinetic data associated to each reaction are expressed with a modified Arrhenius law $k(T)=A \times T^{n} \exp ^{-\frac{E_{a}}{R T}}$ where $T$ is the temperature, $E_{\mathrm{a}}$ the activation energy of the reaction, $A$ the pre-exponential factor, and $n$ a coefficient that allows the temperature dependence of the pre-exponential factor. If the rate constant associated to the forward reaction is $k_{f i}(T)$, then the one associated to the reverse reaction is $k_{r i}(T)$, verifying

$K_{p i}=\frac{k_{f i}(T)}{k_{r i}(T)}\left(\frac{k_{\mathrm{B}} T}{P^{0}}\right)^{\sum_{l=1}^{L} v_{l i}}$

where $K_{p i}$ is the equilibrium constant, when the activity of the reactants is expressed in pressure units (Benson 1976):

$$
K_{p i}=\exp \left(\frac{\Delta S_{i}^{0}}{R}-\frac{\Delta H_{i}^{0}}{R T}\right)
$$

\footnotetext{
${ }^{8}$ http://www.ca.sandia.gov/HiTempThermo/index.hml, http://garfield.chem.elte.hu/Burcat/burcat.html4
}

Here, $\Delta S_{i}^{0}$ and $\Delta H_{i}^{0}$ are the variation in entropy and enthalpy occurring when passing from reactants to products in the reaction $i, P^{0}$ is the standard pressure $\left(P^{0}=1.01325 \mathrm{bar}\right), k_{\mathrm{B}}$ is the Boltzmann's constant, and $v_{l}$ are the stoichiometric coefficients of the $L$ species involved in reaction $i: v_{l}=v_{l i}^{\prime \prime}-v_{l i}^{\prime}$. Combined with Eqs. (A.2) and (A.3), $\frac{\Delta S_{i}^{0}}{R}$ and $\frac{\Delta H_{i}^{0}}{R T}$ can be calculated with the NASA coefficients:

$$
\frac{\Delta S_{i}^{0}}{R}=\sum_{l=1}^{L} v_{l} \frac{s_{l}^{0}(T)}{R} \quad \text { and } \quad \frac{\Delta H_{i}^{0}}{R T}=\sum_{l=1}^{L} v_{l} \frac{h_{l}^{0}(T)}{R T}
$$

Finally, we can calculate the reverse reaction rate for the reaction $i$ :

$k_{r i}(T)=\frac{k_{f i}(T)}{K_{p i}}\left(\frac{k_{\mathrm{B}} T}{P^{0}}\right)^{\sum_{l=1}^{L} v_{l i}}$.

\section{Appendix B: Chemical equilibrium calculation}

To compute the equilibrium abundance of the species in a definite system considered as an ideal gas, we have developed a thermodynamical equilibrium calculator TECA. TECA is software that allows equilibrium calculation for a complex mixture. More specifically, for a given initial state of an ideal-gas mixture, the chemical-equilibrium program is able to determine the gas composition at a defined temperature and pressure. This calculation is based on the principle of the minimization of Gibbs energy (e.g. Gibbs 1873; White et al. 1958; Eriksson \& Rosen 1971; Smith \& Missen 1982; Reynolds 1986):

$G=\sum_{l=1}^{L} \overline{g_{l}} N_{l}$

where $L$ is the total number of species, $\overline{g_{l}}$ the partial free energy of the species $l$, and $N_{l}$ the number of moles of the species $l$.

The partial free energy of a compound $l$, behaving as an ideal gas, is given by

$\overline{g_{l}}=g_{l}(T, P)+R T \ln N_{l}$

where $g_{l}(T, P)$ is the free energy of the species $l$ at the temperature $T$ and the pressure $P$ of the system and $R$ is the ideal gas constant.

For an ideal gas, $g_{l}(T, P)$ is given by

$g_{l}(T, P)=h_{l}^{0}(T)-T s_{l}^{0}(T)+R T \ln \left(\frac{P}{P^{0}}\right)$

where $h_{l}^{0}(T)$ and $s_{l}^{0}(T)$ are respectively, the standard-state enthalpy and entropy of the species $l$ at the temperature $T$ of the system.

The enthalpy and the entropy are expressed as NASA polynomials as described above.

\section{Appendix C: Pressure-dependent reactions}

Under some conditions, several reactions do not have the same rate constant depending on whether they occur under low or high pressure (respectively $k_{0}(T)$ and $k_{\infty}(T)$ ). In this case, between these two limits what is called a fall-off zone appears. This is typically the case in reactions requiring a collisional body to proceed, such as thermal dissociation or recombination (threebody) reactions. In the present kinetic model, we have different types of reactions with pressure-dependent rate constants (Table C.1). In some cases, some species act more efficiently 
Table C.1. Some examples of reactions with pressure-dependent rate constants present in the kinetic model.

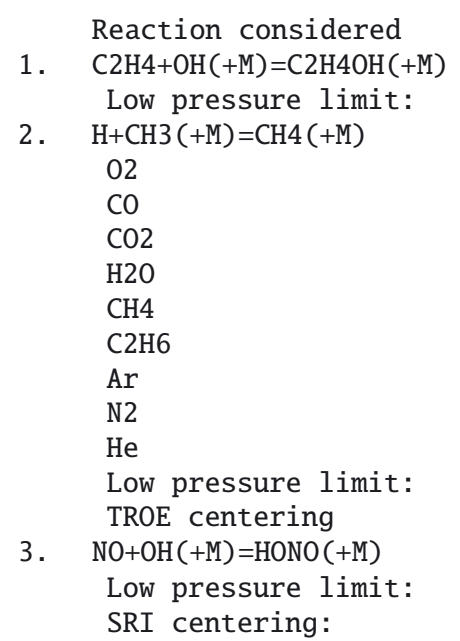

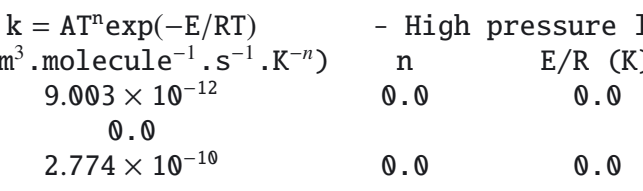

0.40

0.75

1.50

6.50

$0.00^{9}$

3.00

Q. 35

0.40

Q. 35

$-1.8$

3315

$\begin{array}{cc}6.484 \times 10^{-25} & -2.4 \\ 1.0 & 0.0\end{array}$ as collisional bodies than do others. Then, when available from the literature, collisional efficiencies are used to specify the increased efficiency of the $l$ th species in the $i$ th reaction (see for example reaction (2) in Table C.1).

For the pressure-dependent reactions, the rate constant at any pressure is taken to be

$k(T)=k_{\infty}(T)\left(\frac{P_{r}}{1+P_{r}}\right) F$

where the reduced pressure $P_{r}$ is given by

$P_{r}=\frac{[M] k_{0}}{k_{\infty}}$

and $[M]$ is the concentration of the mixture, weighted by the efficiency of each compound, $\alpha_{l}$, in the reaction studied:

$[M]=\sum_{l=1}^{L} \alpha_{l}\left[X_{l}\right]$

where $\left[X_{l}\right]$ is the concentration of the species $k$.

As shown in Table C.1, three methods of representation of the rate expression in the fall-off region are used (enhanced collisional body efficiencies of certain species are presented below the reaction):

- the Lindemann et al. (1922) formulation, illustrated by reaction (1) in Table C.1;

- the Troe (1983) formulation, see for example reaction (2) in Table C.1;

- the SRI formulation proposed by Stewart et al. (1989), illustrated by reaction (3) in Table C.1.

In the Lindenman form, $F$ is unity $(F=1)$.
In the Troe form $F$ is given by

$\log _{10} F=\frac{\log _{10}\left(F_{\text {cent }}\right)}{1+\left[\frac{\log _{10}\left(P_{r}\right)+c}{N-d\left(\log _{10}\left(P_{r}\right)+c\right)}\right]^{2}}$

with

$c=-0.4-0.67 \times \log _{10}\left(F_{\text {cent }}\right)$

$N=0.75-1.27 \times \log _{10}\left(F_{\text {cent }}\right)$

$d=0.14$

and

$F_{\text {cent }}=(1-a) \exp \left(-\frac{T}{T^{* * *}}\right)+a \exp \left(-\frac{T}{T^{*}}\right)+\exp \left(-\frac{T^{* *}}{T}\right)$

the four parameters $a, T^{* * *}, T^{*}$ and $T^{* *}$ must be specified but it is often the case that the parameter $T^{* *}$ is not used because of the lack of data.

The approach taken at the Stanford Research Institute (SRI) by Stewart et al. (1989) is in many ways similar to that taken by Troe, but the blending function $F$ is approximated differently. Here, $F$ is given by

$F=d\left[a \exp \frac{-b}{T}+\exp \frac{-T}{c}\right]^{X} T^{e}$,

where

$X=\frac{1}{1+\left(\log _{10} P_{r}\right)^{2}}$. 
O. Venot et al.: A chemical model for the atmosphere of hot Jupiters

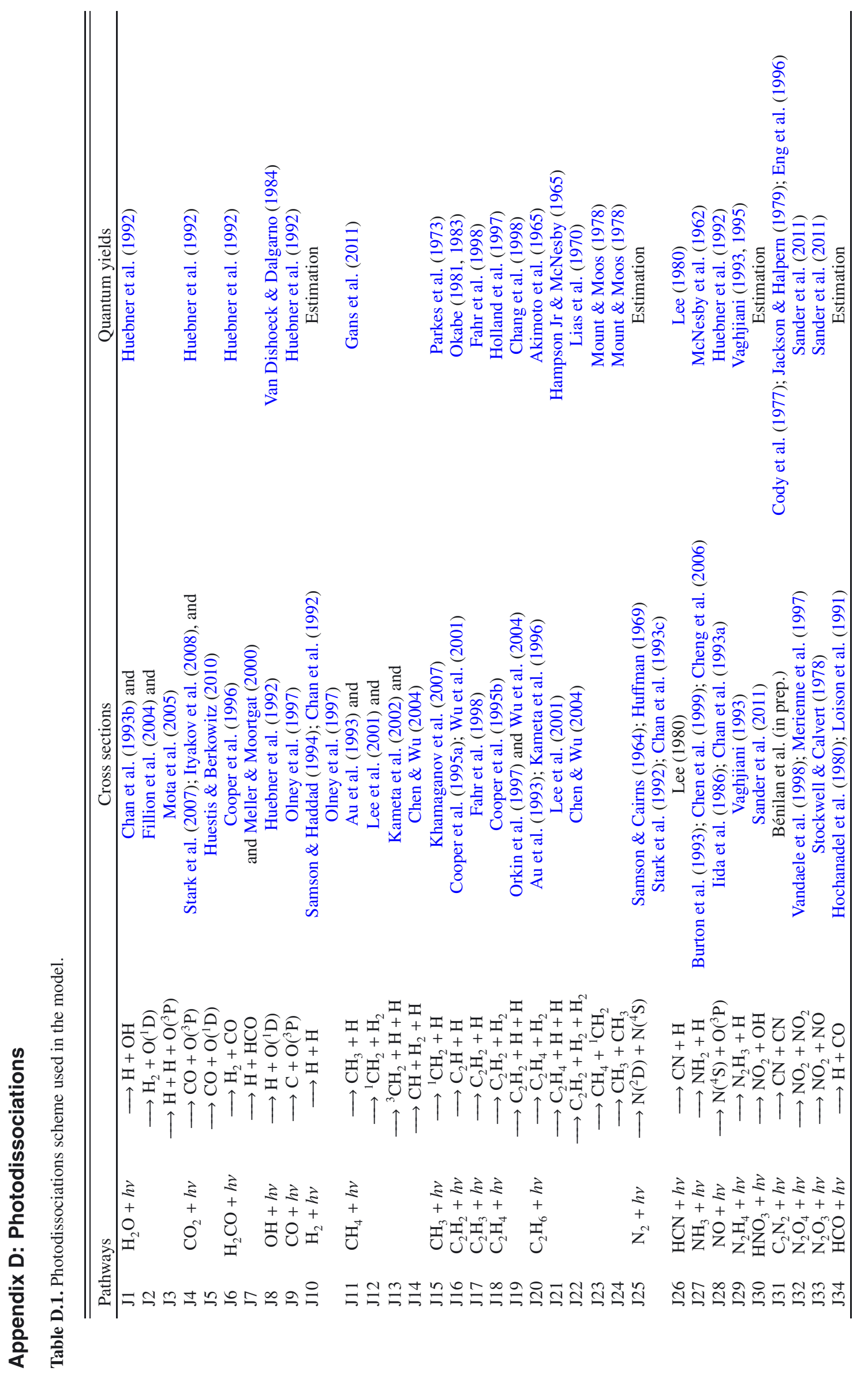

